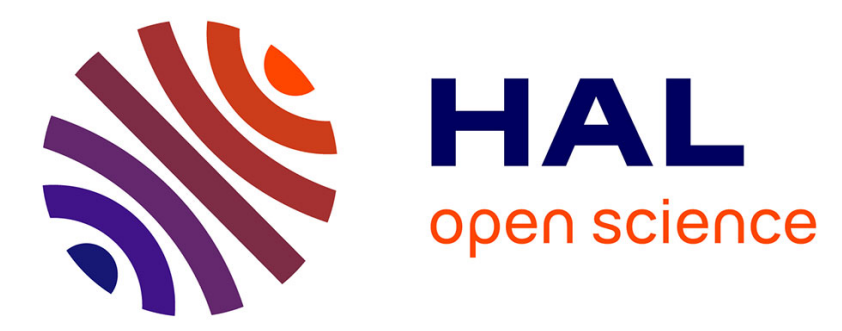

\title{
Mathematical investigation of innate immune responses to lung cancer: The role of macrophages with mixed phenotypes
}

\author{
Raluca Eftimie, Charlotte Barelle
}

\section{- To cite this version:}

Raluca Eftimie, Charlotte Barelle. Mathematical investigation of innate immune responses to lung cancer: The role of macrophages with mixed phenotypes. Journal of Theoretical Biology, 2021, 524, pp.110739. 10.1016/j.jtbi.2021.110739 . hal-03258055

\section{HAL Id: hal-03258055 \\ https://hal.science/hal-03258055}

Submitted on 11 Jun 2021

HAL is a multi-disciplinary open access archive for the deposit and dissemination of scientific research documents, whether they are published or not. The documents may come from teaching and research institutions in France or abroad, or from public or private research centers.
L'archive ouverte pluridisciplinaire HAL, est destinée au dépôt et à la diffusion de documents scientifiques de niveau recherche, publiés ou non, émanant des établissements d'enseignement et de recherche français ou étrangers, des laboratoires publics ou privés. 


\title{
Mathematical investigation of innate immune responses to lung cancer: the role of macrophages with mixed phenotypes
}

\author{
Raluca Eftimie ${ }^{\mathrm{a}, \mathrm{b}, *}$, Charlotte Barelle ${ }^{\mathrm{a}}$ \\ ${ }^{a}$ Division of Mathematics, University of Dundee, Dundee, United Kingdom, DD1 $4 H N$ \\ ${ }^{b}$ Laboratoire Mathématiques de Besançon, UMR - CNRS 6623, Université de \\ Bourgogne Franche-Comté, 25000 Besançon, France
}

\begin{abstract}
Macrophages' role in the evolution of solid tumours is a well accepted fact, with the M1-like macrophages having an anti-tumour role and the M2-like macrophages having a pro-tumour role. Despite the fact that some clinical studies on lung tumours have emphasised also the presence of macrophages with mixed M1 and M2 phenotypes in addition to macrophages with distinct phenotypes, the majority of studies still use the distinct M1-M2 classification to predict the evolution of tumours and patient survival. In this theoretical study we use a mathematical modelling and computational approach to investigate the role of macrophages with mixed phenotype on growth/control/elimination of lung tumours. We show that tumour control in the presence of M2 $\rightarrow$ M1 re-polarising treatments is mainly the result of macrophages with mixed phenotypes (due to the assumption of short half-life of M1-like macrophages). We also show that the half-life of various macrophage phenotypes (distinct M1 or mixed M1/M2 phenotypes) impacts the outcome of various therapeutic strategies targeting tumour-associated macrophages. All these results suggest the need for a better experimental understanding of the kinetics of macrophages inside solid tumours.
\end{abstract}

Keywords: Mathematical modelling; Non-small cell lung cancer; M1 and M2 macrophages; Macrophages with mixed phenotypes;

2020 MSC: $92 \mathrm{C} 50$

\section{Introduction}

The non-small cell lung cancer is the most common type of lung cancers, and the leading cause of cancer-related deaths in the world [1, 2]. Due to the

\footnotetext{
* Corresponding author

Email address: r.a.eftimie@dundee.ac.uk; raluca.eftimie@univ-fcomte.fr (Raluca Eftimie )
}

Preprint submitted to J. Theor. Biol.

April 21, 2021 
absence of clinical symptoms, most cancers are diagnosed only when they reach an advanced stage and when treatments are not effective anymore. For a long time it was thought that NSCLC is non-immunogenic, but over the last decade a number of studies have shown that this lack of immunity is the result of immuneevasive mechanisms employed by the tumour cells [1]. A recent study [3] on the immune cell composition of human NSCLC has shown that $\mathrm{T}$ cells represent the most abundant immune cell population $(\approx 47 \%)$, followed by B cells $(\approx 16 \%)$, macrophages $(\approx 4.7 \%)$ and $\mathrm{NK}$ cells $(\approx 4.5 \%)$. However, in 4 ] the authors found that neutrophils are the most prevalent immune cells in NSCLC, while in 5 the authors identified more macrophages than $\mathrm{T}$ cells inside tumour islets and stroma. A very recent study [6] that analysed publicly available raw microarray expression data on immune composition of NSCLC concluded that the majority of the immune infiltrates inside these tumours is represented by the macrophages, followed by $\mathrm{T}$ cells and $\mathrm{B}$ cells. Moreover, in regard to the infiltrating macrophages, the authors in [6] concluded that the majority of these cells have a M2-like phenotype (i.e., alternatively activated, anti-inflammatory and pro-tumour cells [7), with the next abundant cells having M1-like phenotypes (i.e., classically activated, pro-inflammatory and anti-tumour cells [7]) or M0 (non-activated) phenotypes. It is possible that some of these contradictory results might be the result of the type of cell markers used to classify the cells, including the different types of macrophages, as discussed below in more detail.

Because many clinical studies [8, 9, 10, 11, 12, have focused on the prognostic value of the numbers/percentages of various macrophage sub-populations that infiltrate various tumour areas (islets, stroma) in NSCLC patients, in this study we focus on the role of macrophages on the growth, control, and elimination of lung tumours. Again, we need to emphasise that all these clinical studies on NSCLC show different results, some being reproduced and summarised in Figure 1. One reason for these differences could be the various markers used to classify the various cell types; e.g., CD68/iNOS [9, 11] and CD68/HLA-DR [8, 9] for M1 cells; CD68/CD163 [8, 9, 11], CD68/CD204 [12, CD68/CD206 13. for M2 cells). In addition, a number of studies that profiled human NSCLC showed that a small percentage of macrophages (i.e., between 0-11\%) have markers characterising M2 cells as well as markers characterising M1 cells [8, 9, 12]; see also Figure 1(a),(b). However, in a very recent study [14] on early-stage lung cancer the authors showed experimentally that tumourassociated macrophages from NSCLC expressed both M1 and M2 markers (e.g. HLA-DR, CD206, CD163), sometimes at levels higher than the in vitro differentiated M1 and M2 macrophages. They also showed that approximately $40 \%$ of $\mathrm{CD}_{14}{ }^{+}$cells identified inside the NSCLC tissue had high levels of both HLADR and CD163 markers, and more than 50\% had high levels of both HLA-DR and CD206 markers. (Note that $\mathrm{CD} 14^{+}$is a human protein produced mostly by macrophages.) Returning to Figure 1, we also note that since some of the markers can be expressed by both M1 and M2 cells, the percentages shown in some of the sub-panels, e.g., sub-panel (b), do not add up to $100 \%$. We can conclude from here that there are still many contradictory results about the proportion of macrophages with mixed phenotypes inside lung tumours (probably due to 

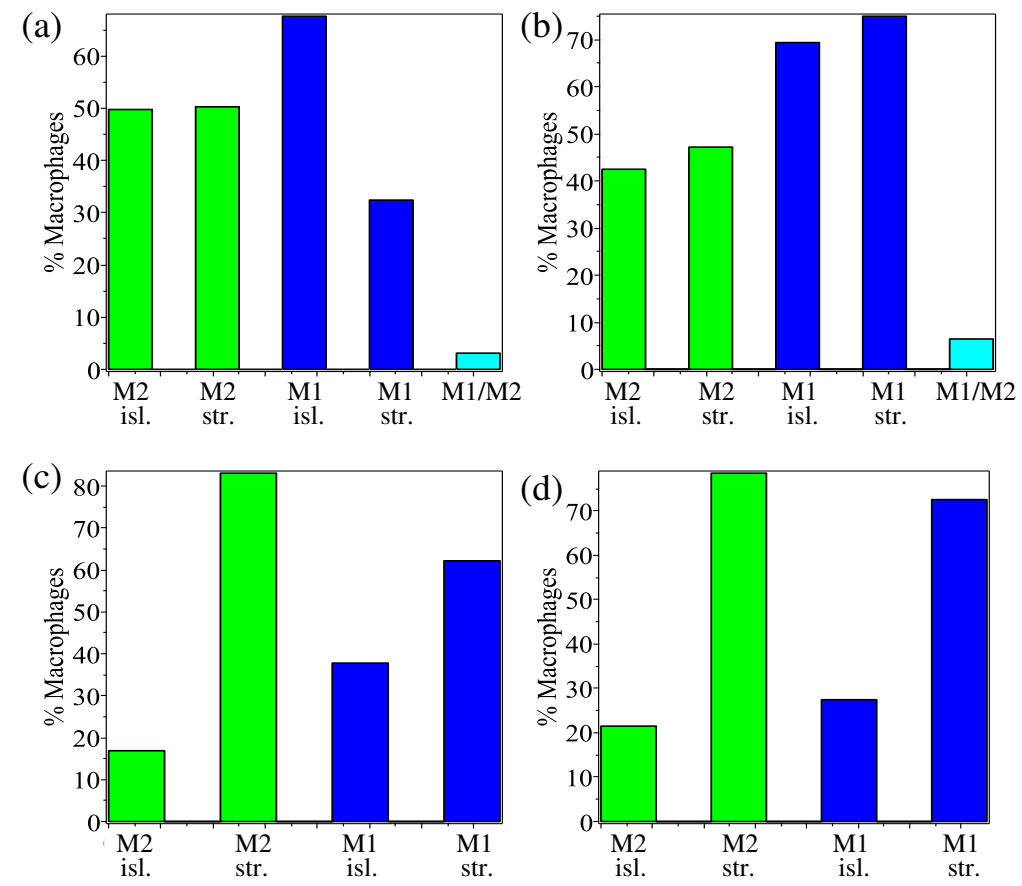

Figure 1: Data on macrophage percentages in tumour islets (isl.) and stroma (str.) as we approximated it from the following clinical studies on NSCLC: (a) the study in 8 . Here we show data for M1 (blue) and M2 (green) percentages in long-term patients survival - to compare it with the study in 9 . Percentages were calculated using the data from Table 2 in 8, by taking the ratio of M1 (or M2) cell numbers in either islets or stroma to the total number of M1 (or M2) cells inside both islets and stroma. The authors also mentioned the existence of a small percentage of cells with mixed M1/M2 phenotype (between $1.2 \%-8.1 \%$ with a median of $3.1 \%$; see the cyan-coloured bar). (b) the study in 9. Here we show data for long-term patients survival. Percentages were taken directly from Table 2 in 9 . The authors also mentioned the existence of a small percentage of cells with mixed M1/M2 phenotype (between $2.5 \%-10.2 \%$ with a median of $6.45 \%$; see the cyan-coloured bar). (c) the study in 10, where there was no mention on whether the data was for long-term or short-term patients. Percentages were taken directly from Table 2 in [10]; (d) the study in [11, where again there was no mention on whether the data was for long-term or short-term patients. We calculated the percentages using the data from Table 3 in [11, by taking the ratio of the total number of M1 (or M2) cells (from patients with stage IIA-IIB cancers) in either islets or stroma over the total number of M1 (or M2) cells inside both islets and stroma. Similar results were obtained for macrophage data from patients with stage IA-IB or IIIA-IIIB cancers.

the fact that the classification of macrophages subsets is still in its infancy [15] and still poses many challenges [16).

To address various questions related to tumour-macrophage interactions (as well as questions about the effect of different immuno- and chemo-therapies on tumour-macrophage interactions), the last few decades have seen the development of a large variety of mathematical models [17, 18, 19, 20, 21, 22, 23, 24. We note that all these models focus on the two extreme types of macrophages, 
2. Model Description

The following mathematical model is used to describe and investigate the interactions between tumour cells and macrophages. This model focuses on the temporal evolution of: tumour cells $\left(u_{T}\right)$, macrophages with a M1-like phenotype $\left(u_{M 1}\right)$, macrophages with a M2-like phenotype $\left(u_{M 2}\right)$, and macrophages 


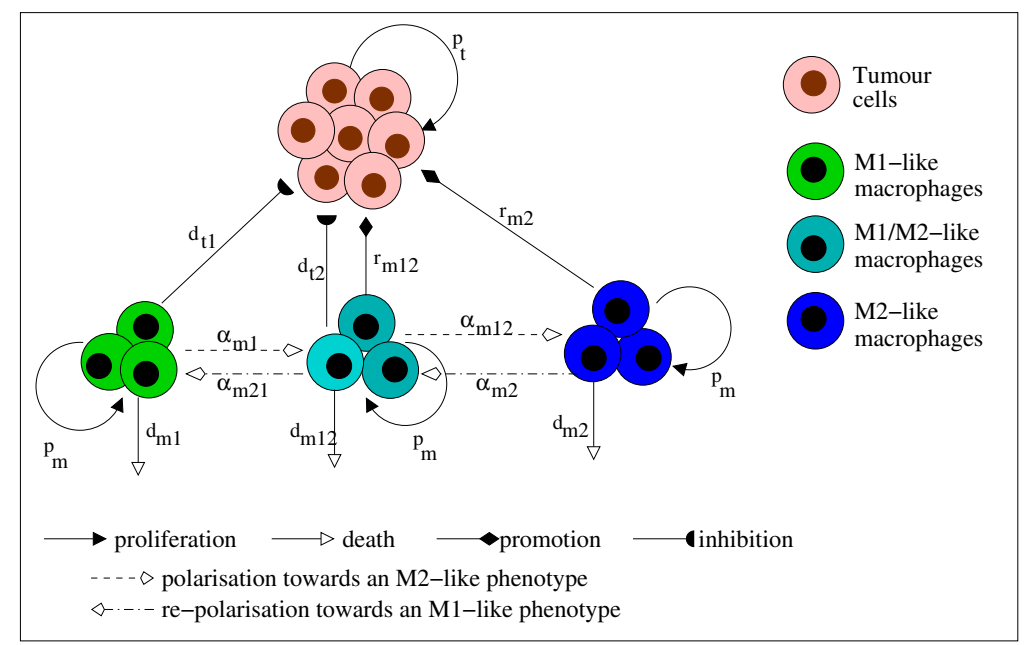

Figure 2: Caricature description of the cell-cell interactions depicted by model (1).

with a mixed M1-M2 phenotype $\left(u_{M 12}\right)$; see also Figure 2.

$$
\begin{aligned}
\frac{d u_{T}}{d t}= & p_{t} u_{T}\left(1-\frac{u_{T}}{K_{t}}\right)\left(1+r_{m 2} u_{M 2}+r_{m 12} u_{M 12}\right)-d_{t 1} u_{T} u_{M 1}-d_{t 2} u_{T} u_{M 12} \\
\frac{d u_{M 1}}{d t}= & p_{m 1} u_{M 1}\left(1-\frac{u_{M 1}+u_{M 12}+u_{M 2}}{K_{m}}\right)-d_{m 1} u_{M 1}-\alpha_{m 1} u_{M 1} \frac{u_{T}}{u_{T}+K_{t}^{*}} \\
& +\alpha_{m 21} u_{M 12}, \\
\frac{d u_{M 12}}{d t}= & p_{m 12} u_{M 12}\left(1-\frac{u_{M 1}+u_{M 12}+u_{M 2}}{K_{m}}\right)-d_{m 12} u_{M 12}+\alpha_{m 1} u_{M 1} \frac{u_{T}}{u_{T}+K_{t}^{*}} \\
& -\alpha_{m 12} u_{M 12} \frac{u_{T}}{u_{T}+K_{t}^{*}}-\alpha_{m 21} u_{M 12}+\alpha_{m 2} u_{M 2}, \\
\frac{d u_{M 2}}{d t}= & p_{m 2} u_{M 2}\left(1-\frac{u_{M 1}+u_{M 12}+u_{M 2}}{K_{m}}\right)-d_{m 2} u_{M 2}+\alpha_{m 12} u_{M 12} \frac{u_{T}}{u_{T}+K_{t}^{*}} \\
& -\alpha_{m 2} u_{M 2} .
\end{aligned}
$$

The above equations incorporate the following biological assumptions:

- Equation (1a) describes the dynamics of tumour cells, which are assumed to grow logistically at a rate $p_{t}$ up to a carrying capacity $K_{T}$. This logistic growth models the phenomenological observation that tumour growth slows down as size increases, due to lack of nutrients [25]. The macrophages with a M2-like phenotype $\left(u_{M 2}\right.$ and $\left.u_{M 12}\right)$ contribute to the proliferation of tumour cells [26, 7, 16, which we model using coefficients $r_{m 2}$ and $r_{m 12}$. The macrophages with a M1-like phenotype reduce tumour growth [7] at rates $d_{t 1}$ (the $u_{M 1}$ cells) and $d_{t 2}$ (the $u_{M 12}$ cells). 
- Equation (1b) describes the dynamics of macrophages with a dominant M1 phenotype $\left(u_{M 1}\right)$. Since tissue-resident macrophages are maintained via self-proliferation with minimal monocyte input [27, 28, 29], and since in [30] the authors showed that macrophages have an exponential growth followed by a stationary phase, here we consider a logistic growth at a rate $p_{m 1}$, up to a carrying capacity $K_{m}$. These cells die naturally 31] at a rate $d_{m 1}$. In the presence of tumour cells, the M1 macrophages can polarise (at a rate $\alpha_{m 1}$ ) towards a mixed M1/M2 phenotype. External factors (e.g., anti-tumour treatments [32] can re-polarise (at a rate $\alpha_{m 21}$ ) the macrophages with a mixed M1/M2 phenotype towards an anti-tumour M1-dominant phenotype.

- Equation (1c) describes the dynamics of macrophages with mixed M1/M2 phenotype $\left(u_{M 12}\right)$. As before, since tissue-resident macrophages are selfproliferating [27, 28] and their proliferation has an exponential phase followed by a stationary phase [30, here we assume a logistic growth at a rate $p_{m 12}$, up to a carrying capacity $K_{m}$. Moreover, these cells die naturally at a rate $d_{m 12}$. Finally, we assume that these cells with mixed M1/M2 phenotypes can polarise (at a rate $\alpha_{m 12}$ ) in the presence of tumour cells towards a more distinct M2 phenotype, or can re-polarise (at a rate $\alpha_{m 21}$ ) following external treatment [32] towards an M1 phenotype. In the presence of tumour cells, M1 macrophages can polarise (at a rate $\alpha_{m 1}$ ) towards a mixed M1/M2 phenotype. Anti-tumour treatments [32] can re-polarise (at a rate $\alpha_{m 2}$ ) the M2 macrophages towards a mixed M1/M2 phenotype.

- Equation (1d) describes the dynamics of macrophages with a dominant M2 phenotype $\left(u_{M 2}\right)$. Again, we assume that this population grows logistically at a rate $p_{m 2}$, up to a carrying capacity $K_{m}$. These cells die naturally at a rate $d_{m 2}$ [31]. Anti-tumour treatments 32] can re-polarise the M2 macrophages (at a rate $\alpha_{m 2}$ ) towards a mixed M1/M2 phenotype. In the presence of tumour cells, the macrophages with a mixed M1/M2 phenotype can polarise (at a rate $\alpha_{m 12}$ ) towards M2 cells.

\subsection{Parameter estimation}

Even if our biological questions were triggered by human NSCLC data (see Figure 11), to identify some of the model parameters we focus on murine experiments for which there is more data (compared to humans). Below we summarise the data we used to estimate different model parameters.

- The study in [33] investigated the growth of tumours resembling nonsmall cell lung cancer (NSCLC) in mice lungs. In Figure 3 we reproduce the murine tumour growth data from [33, together with the solution of a logistic equation for tumour growth. The best fit of the numerical solution to the data (obtained using the classical least square method) was obtained for $p_{t}=0.23$ and $K_{t}=1400$. This proliferation rate is consistent with the doubling time (i.e., 2.97 days) of NSCLC cells inoculated into nude mice, as calculated experimentally in 34 . 

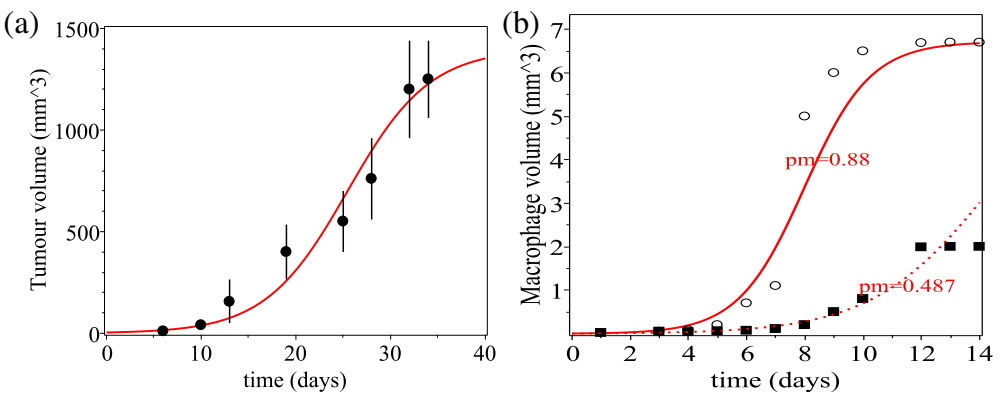

Figure 3: (a) Reproduction of tumour growth data from 33 (black circles) together with the solution of a logistic growth equation for tumour growth (red curve), as given by eq. 1a) in the absence of any macrophages. Here $K_{t}=1400$ and $p_{t}=0.23$, and the initial condition for the numerical simulation of the tumour logistic growth is $u_{T}(0)=4 \mathrm{~mm}^{3}$. (b) Reproduction of macrophage growth data from [30] (open circles and black squares) - where data was transformed from number of cells to potential volume occupied by these cells (see discussion below) - together with the solution of a logistic equation for macrophage growth (red curves), as given by the sums of of eqns. (1b) $+1 \mathrm{c})+(1 \mathrm{~d})$ when $p_{m 1}=p_{m 12}=p_{m 2}:=p_{m}$ and $d_{m 1}=d_{m 12}=d_{m 2}=0$. The continuous curve was obtained for $p_{m}=0.88$ and $K_{m}=6.72$, while the dotted curve was obtained for $p_{m}=0.483$ and $K_{m}=6.72$. The initial condition for the numerical simulation of macrophage logistic equation is $u_{M}(0)=0.006 \mathrm{~mm}^{3}$ (where $u_{M}$ describes the total macrophage size).

- In 3 the authors have calculated that macrophages represent $\approx 4.8 \%$ of the total immune infiltrates into human NSCLC. For our murine model, we assume that immune cell infiltrates represent up to $10 \%$ of tumour mass, and the macrophages represent $4.8 \%$ of these immune infiltrates. (Note that in $[10$ it was estimated that macrophages represent $\approx 15.84 \%$ of all cells inside tumour tissue, and thus our assumption is not completely unrealistic.) Thus, for a maximum tumour volume of $K_{t}=1400 \mathrm{~mm}^{3}$ we obtain a maximum macrophage volume of $K_{m}=6.72 \mathrm{~mm}^{3}$.

- In 35 the authors calculated the diameter of an alveolar macrophage at $\approx 19 \mu \mathrm{m}$. In [36] the author suggested that a volume of $1000 \mathrm{~mm}^{3}$ can contain up to $9.39 \times 10^{7}$ cells of diameter $22 \mu \mathrm{m}$, or up to $2.44 \times 10^{8}$ cells of diameter $16 \mu \mathrm{m}$. In this study, we assume that a volume of $1000 \mathrm{~mm}^{3}$ can contain $\approx 10^{8}$ macrophages. Thus a carrying capacity $K_{m}=6.72 \mathrm{~mm}^{3}$ can contain $\approx 6.72 \times 10^{5}$ macrophages. These numbers are consistent with the experimental study in [37, where the authors showed that the number of macrophages from control mice ranged from $8 \times 10^{4}$ cells/mouse to $2.4 \times 10^{5}$ cells $/$ mice.

In [30], the authors measured macrophage growth, and calculated a proliferation rate between 0.487 /day and 0.88 /day (in different mice). In Figure 3 we approximated the two macrophage data sets from 30] (Fig. 14.20.1 in 30, which shows cell numbers), where we transformed cell numbers into cell volumes (using the assumptions and calculations above). Since in [30] the authors showed that macrophages grow logistically, we 
fitted a logistic growth curve with rate $p_{m}$ and carrying capacity $K_{m}=$ $6.72 \mathrm{~mm}^{3}$. The continuous red curve in Figure 3 shows macrophage growth for $p_{m}=0.88$, while the dashed red curve shows macrophage growth for $p_{m}=0.487$. Throughout this study we will consider an average macrophage proliferation rate of $p_{m 1}=p_{m 12}=p_{m 2}=0.7$.

- The experimental study in [27, which focused on the adoptive transfer of human mononuclear phagocytes into mice, showed that classical (M1) macrophages circulate for a mean of 1.01 days, whereas intermediate (M12) and nonclassical (M2) macrophages have longer mean lifespans of 4.30 and 7.41 days, respectively. In 31 the authors stated that the murine M1-like macrophages (involved in phagocytosis) have a half-life between 18-20hrs, while the murine M2-like macrophages (involved in tissue repair) have a half-life between 5-7 days. Therefore, in this study we assume that:

- the M1-like macrophages have a half-life of $\approx 0.8$ days (i.e., $\approx 19.2 \mathrm{hr}$ ), corresponding to a death rate $d_{m 1}=0.87 /$ day;

- the mixed M1/M2 macrophages have a half-life of $\approx 3$ days, corresponding to a death rate $d_{m 12}=0.23 /$ day;

- the M2-like macrophages have a half-life of $\approx 5.14$ days, corresponding to a death rate $d_{m 2}=0.09 /$ day.

All other parameter values that appear in model (1) are unknown. In the following, we discuss some of the assumptions we consider when choosing the ranges over which we vary these parameters:

- We assume that the presence of $1 \%$ of max tumour is enough to trigger a $u_{M 1} \rightarrow u_{M 12}$ polarisation, and further a $u_{M 12} \rightarrow u_{M 2}$ polarisation. Thus, we consider $K_{t}^{*}=1 \% K_{t}$.

- We assume that macrophages with mixed M1/M2 phenotypes exhibit half the anti-tumour effect of the M1 macrophages. Thus, we consider $d_{t 1} \in$ $\left(10^{-5}, 10^{-1}\right)$ (with a baseline value of $\left.d_{t 1}=0.01\right)$ and $d_{t 2} \in\left(5 \times 10^{-6}, 5 \times\right.$ $10^{-2}$ ) (with a baseline value of $d_{t 2}=0.005$ ).

- We assume that macrophages with mixed M1/M2 phenotype have half the pro-tumour effect of M2 macrophages. Thus, we consider $r_{m 2} \in\left(10^{-2}-\right.$ $\left.10^{0}\right)$ (with a baseline value $\left.r_{m 2}=0.1\right)$ and $r_{m 12} \in\left(5 \times 10^{-3}, 0.5\right)$ (with a baseline value $\left.r_{m 12}=0.05\right)$.

- In humanised murine experiments [27] it has been found that that only a small proportion of M1 macrophages will re-polarise to an intermediate M12 phenotype, but most M12 macrophages will re-polarise to an M2 phenotype during their lifespan. Throughout this study we assume $\alpha_{m 1}<\alpha_{m 12}$. For the re-polarisation rates we assume $\alpha_{m 2}=\alpha_{m 21}$. Thus, we consider $\alpha_{m 1} \in\left(10^{-4}, 10^{-1}\right) /$ day (with a baseline $\alpha_{m 1}=10^{-3} /$ day), $\alpha_{m 12} \in\left(10^{-3}, 10^{-1}\right) /$ day (with a baseline of $\alpha_{m 12}=10^{-2} /$ day), and 
$\alpha_{m 2}=\alpha_{m 21} \in\left(10^{-3}, 10^{0}\right) /$ day (with baselines of 0.0 , since we assume that initially there is no treatment to force a macrophage re-polarisation towards an M1-like phenotype).

All these estimated parameter values and ranges which appear in model (1) are summarised in Table 2.1. For simplicity, and to avoid numerical problems caused by a stiff system, we rescale the tumour cells by $K_{t}$ and the macrophages by $K_{m}$. This leads to unitary carrying capacities $\left(\overline{K_{t}}=1, \overline{K_{m}}=1\right)$, and to a rescaling of the following five parameters:

$$
\begin{aligned}
& \overline{d_{t 1}}=d_{t 1} \times K_{m}, \quad \overline{d_{t 2}}=d_{t 2} \times K_{m}, \quad \overline{r_{m 2}}=r_{m 2} \times K_{m}, \\
& \overline{r_{m 12}}=r_{m 12} \times K_{m}, \quad \overline{K_{t}^{*}}=\frac{K_{t}^{*}}{K_{t}} .
\end{aligned}
$$

These rescaled parameter values are listed in the fourth column in Table 2.1 - where we removed the bars for simplicity. These values are used for the numerical simulations performed throughout this study.

\section{Numerical results}

The initial conditions for the numerical simulations of system (1) were obtained from the rescaled initial conditions in Figure 3

$$
\begin{aligned}
& u_{T}(0)=\frac{4}{K_{t}}=\frac{4}{1400}=0.002857, \\
& u_{M 1}(0)=\frac{0.006}{K_{m}}=\frac{0.006}{6.72}=0.000893, \\
& u_{M 12}(0)=0.0, \quad u_{M 2}(0)=0.0 .
\end{aligned}
$$

Thus, we assume that when the tumour is introduced into the system, it elicits a pro-inflammatory immune response characterised only by the presence of a non-zero $u_{M 1}$ population. The numerical solution is propagated in time using a classical fourth order Runge-Kutta method.

\subsection{Baseline system dynamics}

Figure 4(a) shows the dynamics of model (1) for the baseline parameter values given in Table 2.1. We see that initially $(t<10$ days $)$ the immune response is dominated by macrophages with a M1 phenotype. Then a transient increase in the cells with a mixed M1/M2 phenotype (for $10<t<30$ ) is associated with a reduction in tumour size. Tumour relapse is associated with an increase in the cells with a M2 phenotype (for $t>40$ ). We emphasise here that this baseline case assumes $\alpha_{m 2}=\alpha_{m 21}=0$ (see Table 2.1), i.e., no external immune treatment to induce a re-polarisation of M2-like macrophages towards an M1-like phenotype 32 .

In Figure 4(b) we show the effect of externally-inducing a macrophage repolarisation on model (1); i.e., $\alpha_{m 2}=\alpha_{m 21}=0.01$. We observe a slight reduction in tumour size in the long term (i.e. $t>80$ days), which is associated with 
Table 1: Summary of the parameter values used in this study. The $2^{\text {nd }}$ column shows the dimensional parameters, the $3^{\text {rd }}$ column shows their units, and the $4^{\text {th }}$ column shows in bold the rescaled parameter values; see eq. 2 (If there is no difference between the values in the $2^{\text {nd }}$ and $4^{\text {rd }}$ column, it means that the parameter was not rescaled). For most of the parameter values we show a whole range, with the value inside the parentheses being the baseline value used for the simulations. The time unit is "day", cells are described by cell volume "vol".

\begin{tabular}{|c|c|c|c|c|}
\hline Param. & $\begin{array}{l}\text { Original } \\
\text { values }\end{array}$ & $\begin{array}{l}\text { Original } \\
\text { Units }\end{array}$ & $\begin{array}{l}\text { Rescaled } \\
\text { values }\end{array}$ & Description (original values) \\
\hline$p_{t}$ & 0.23 & $\frac{1}{d a y}$ & 0.23 & Proliferation rate of tumour cells \\
\hline$K_{t}$ & 1400 & vol & 1 & Tumour carrying capacity \\
\hline$K_{t}^{*}$ & 14 & vol & 0.01 & $\begin{array}{l}\text { Tumour level that triggers } \mathrm{M} 1 \rightarrow \mathrm{M} 2 \\
\text { macrophage polarisation }\end{array}$ \\
\hline$r_{m 2}$ & $\begin{array}{l}10^{-2}-10^{0} \\
(0.1)\end{array}$ & $\frac{1}{v o l}$ & $\begin{array}{l}6.72 \times 10^{-2}- \\
6.72(0.672)\end{array}$ & $\begin{array}{l}\text { Contribution of M2 macrophages to } \\
\text { the proliferation of tumour cells }\end{array}$ \\
\hline$r_{m 12}$ & $\begin{array}{l}0.005-0.5 \\
(0.05)\end{array}$ & $\frac{1}{v o l}$ & $\begin{array}{ll}0.0336 & - \\
3.36(0.336)\end{array}$ & $\begin{array}{l}\text { Contribution of macrophages with } \\
\text { mixed M1/M2 phenotype to the } \\
\text { proliferation of tumour cells }\end{array}$ \\
\hline$d_{t 1}$ & $\begin{array}{l}10^{-2}-10^{0} \\
(0.2)\end{array}$ & $\frac{1}{d a y \cdot v o l}$ & $\begin{array}{l}6.72 \times 10^{-2}- \\
6.72(1.344)\end{array}$ & $\begin{array}{l}\text { Tumour killing rate by M1 } \\
\text { macrophages }\end{array}$ \\
\hline$d_{t 2}$ & $\begin{array}{l}5 \times 10^{-3}- \\
0.5(0.1)\end{array}$ & $\frac{1}{d a y \cdot v o l}$ & $\begin{array}{l}3.36 \times 10^{-2}- \\
3.36(0.672)\end{array}$ & $\begin{array}{l}\text { Tumour killing rate by macrophages } \\
\text { with mixed M1/M2 phenotype }\end{array}$ \\
\hline$p_{m 1}$ & $\begin{array}{l}0.487-0.88 \\
(0.7)\end{array}$ & $\frac{1}{d a y}$ & $\begin{array}{l}0.487-0.88 \\
(0.7)\end{array}$ & Proliferation rate of M1 cells \\
\hline$p_{m 12}$ & $\begin{array}{l}0.487-0.88 \\
(0.7)\end{array}$ & $\frac{1}{d a y}$ & $\begin{array}{l}0.487-0.88 \\
(0.7)\end{array}$ & $\begin{array}{l}\text { Proliferation rate of macrophages } \\
\text { with mixed M1/M2 phenotype }\end{array}$ \\
\hline$p_{m 2}$ & $\begin{array}{l}0.487-0.88 \\
(0.7)\end{array}$ & $\frac{1}{d a y}$ & $\begin{array}{l}0.487-0.88 \\
(0.7)\end{array}$ & Proliferation rate of M2 cells \\
\hline$K_{m}$ & 6.72 & vol & 1 & Macrophages carrying capacity \\
\hline$d_{m 1}$ & $\begin{array}{l}0.83-0.924 \\
(0.87)\end{array}$ & $\frac{1}{d a y}$ & $\begin{array}{l}0.83-0.924 \\
(0.87)\end{array}$ & Natural death rate of M1 cells \\
\hline$d_{m 12}$ & $\begin{array}{l}0.14-0.83 \\
(0.23)\end{array}$ & $\frac{1}{d a y}$ & $\begin{array}{l}0.14-0.83 \\
(0.23)\end{array}$ & $\begin{array}{l}\text { Natural death rate of cells with } \\
\text { mixed M1/M2 phenotype }\end{array}$ \\
\hline$d_{m 2}$ & $\begin{array}{l}0.09-0.14 \\
(0.1)\end{array}$ & $\frac{1}{d a y}$ & $\begin{array}{l}0.09-0.14 \\
(0.1)\end{array}$ & Natural death rate of M2 cells \\
\hline$\alpha_{m 1}$ & $\begin{array}{l}10^{-5}-10^{-2} \\
(0.001)\end{array}$ & $\frac{1}{d a y}$ & $\begin{array}{l}10^{-5}-10^{-2} \\
(0.001)\end{array}$ & $\begin{array}{l}\text { Polarisation rate of M1 cells towards } \\
\text { a mixed M1/M2-phenotype }\end{array}$ \\
\hline$\alpha_{m 12}$ & $\begin{array}{l}10^{-4}-10^{-1} \\
(0.01)\end{array}$ & $\frac{1}{d a y}$ & $\begin{array}{l}10^{-4}-10^{-1} \\
(0.01)\end{array}$ & $\begin{array}{l}\text { Polarisation rate of macrophages } \\
\text { with a mixed M1/M2 phenotype to- } \\
\text { wards a M2-dominant phenotype }\end{array}$ \\
\hline$\alpha_{m 2}$ & $0-10^{0}(0.0)$ & $\frac{1}{d a y}$ & $0-10^{0}(0.0)$ & $\begin{array}{l}\text { Re-polarisation rate of M2 cells to- } \\
\text { wards a mixed M1/M2-phenotype }\end{array}$ \\
\hline$\alpha_{m 21}$ & $0-10^{0}(0.0)$ & $\frac{1}{d a y}$ & $0-10^{0}(0.0)$ & $\begin{array}{l}\text { Re-polarisation rate of macrophages } \\
\text { with a mixed M1/M2 phenotype to- } \\
\text { wards a M1-dominant phenotype }\end{array}$ \\
\hline
\end{tabular}


a higher percentage of macrophages with mixed M1/M2 phenotype (and a very small but non-zero population of M1-like macrophages). The effect of further increasing $\alpha_{m 2}, \alpha_{m 21}$ will be discussed below, in Figure $7(\mathrm{~d})$.
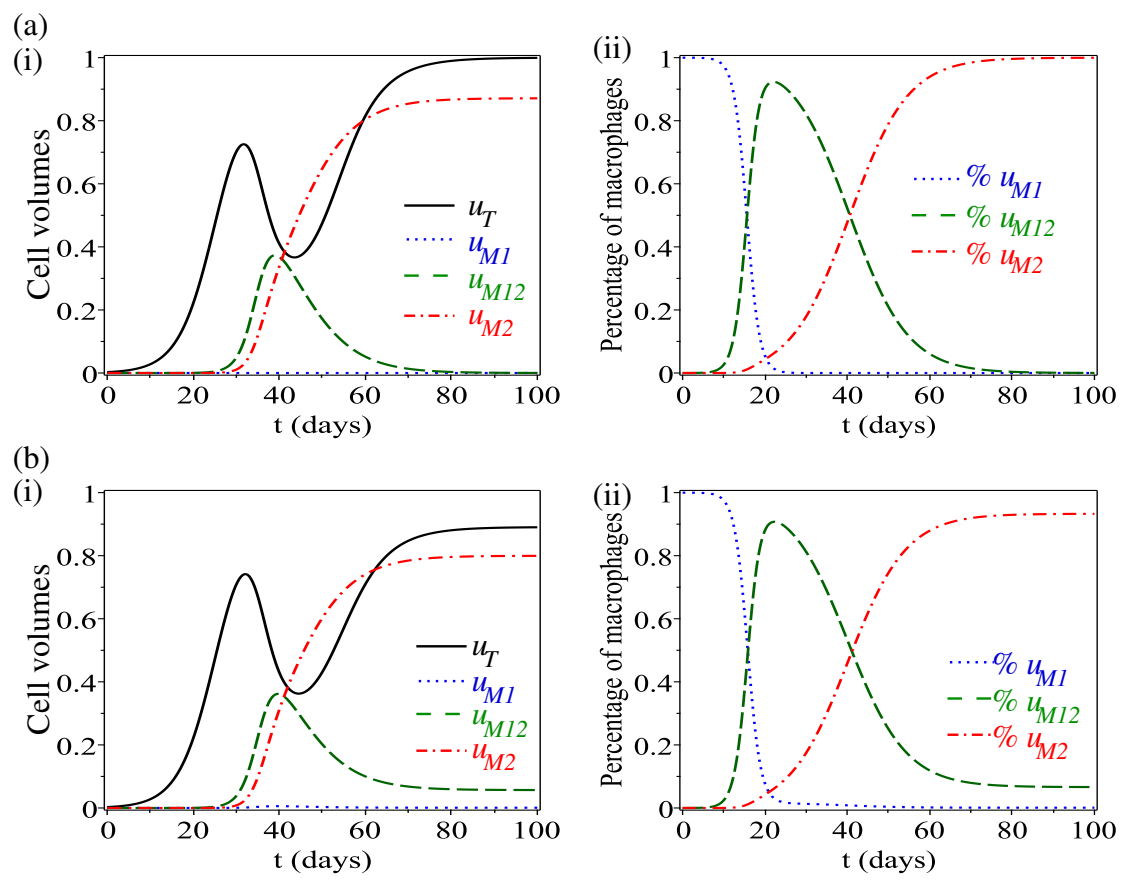

Figure 4: (a) Dynamics of model 11, for the baseline parameter values listed in Table 2.1 when $\alpha_{m 2}=\alpha_{m 21}=0$. (b) Dynamics of model (1), when we assume $\alpha_{m 2}=\alpha_{m 21}=0.01>0$. Sub-panels (i) show the time-evolution of the tumour cells and macrophages; Sub-panels (ii) show the time-evolution of the percentage of macrophage composition.

\subsection{Sensitivity analysis}

Since many parameter values were estimated within certain ranges, in the following we evaluate the robustness of model (1), by investigating the sensitivity of tumour size to small perturbations in some model parameters and in initial conditions. We start in Section 3.2.1 with a local sensitivity analysis to investigate how small perturbations in the initial conditions and macrophage polarisation/re-polarisation rates impact tumour dynamics. However, since we do not know if there is interaction between these polarisation/re-polarisation rates and all other estimated parameters that appear in the model, in Section 3.2 .2 , we perform a global sensitivity analysis.

\subsubsection{Local sensitivity analysis}

In the following we focus on tumour size at $t=40$ (while tumour is decreasing following an increase in macrophages with mixed phenotypes; see Figure 4 and 
at $t=60$ (while tumour relapses and is close to its carrying capacity; see Figure 4). The change in tumour size is calculated using the discrete formula for the derivative of the output (tumour size) with respect to the input (e.g., initial condition $u_{j}(0)$, where $\left.j \in\{T, M 1, M 12, M 2\}\right)$ [38:

$$
L_{S}=\frac{u_{T}^{\text {new }}(40)-u_{T}^{\text {baseline }}(40)}{\left|u_{j}^{\text {new }}(0)-u_{j}^{\text {baseline }}(0)\right|} .
$$

Remark 1. We decided to focus on time $t=60$, when the relapsing tumour would approach the carrying capacity (for the baseline parameter values) since we would like to understand the mechanisms that could impact the late-stage tumours, as many lung tumours are discovered when they have already reached a late stage. Moreover, for this local sensitivity analysis we decided to ignore the earlier times (e.g., $t<30)$ since the immune response was too weak at these times, and changes in immune-related parameters had almost no impact on tumour size. We chose to focus on $t=40$ because at this time the level of immune response (and in particular the macrophages with mixed phenotype) is high enough to have a significant impact on tumour.

Remark 2. In eq. (4) we do not normalise the sensitivity index [39]. This is mainly because when we investigate local sensitivity to the initial conditions, there are two zero baseline initial conditions for $u_{M 2}$ and $u_{M 12}$ and therefore we cannot divide by these values for normalisation (i.e., the ratio $\mid u_{j}^{\text {new }}(0)-$ $u_{j}^{\text {baseline }}(0) \mid / u_{j}^{\text {baseline }}(0), j \in\{M 12, M 2\}$, does not make sense). A similar problem is encountered when we perform the sensitivity to $\alpha_{m 2}$ and $\alpha_{m 21}$ (which have zero baseline values; see Table 2.1). For this reason, in this study we decided to work with equation (4) and not with a normalised version of this equation.

Tumour sensitivity to initial conditions. In Figure 5 we show the magnitude of changes (i.e., $L_{S}$ given by eq. (4)) in tumour size on days (a) $t=40$ and (b) $t=60$, as we increase/decrease the initial conditions for the tumour and M1 macrophage populations by a factor of $10^{2}$ from their baseline values. The M12 and M2 initial macrophage levels are increased from 0 to $10^{-3}$. We see in Figure 5 that the initial conditions for all macrophage sub-populations have a significant impact on the early stages of tumour growth (i.e., $t<50$ ), and only the mixed phenotype macrophages seem to play an important role also in the later stages $(t>50)$. Moreover, increasing the initial tumour size by a factor of $10^{2}$ does not seem to have an impact on either early or later tumour stages, which suggests that the baseline initial tumour size is already large enough. Only a decrease in the initial tumour size has an impact on tumour levels on both $t=40$ and $t=60$.

Tumour sensitivity to polarisation/re-polarisation rates. In Figure 6 we show the magnitude of changes in tumour size on days $t=40$ (panel (a)) and $t=60$ 

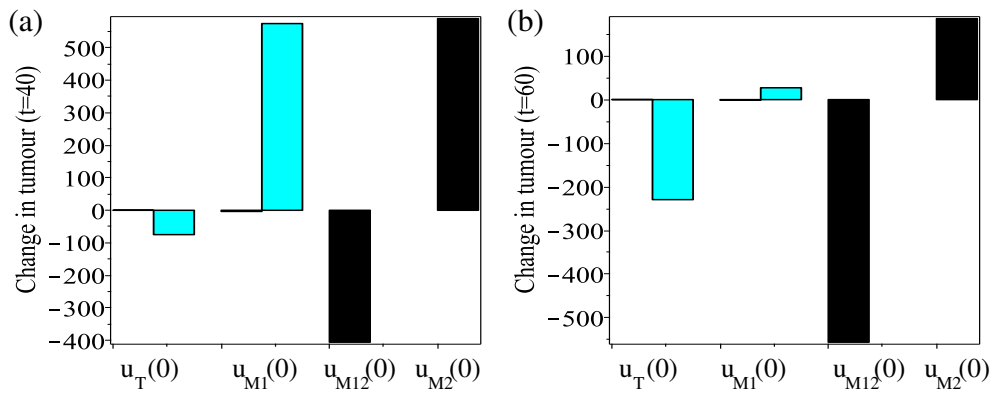

\begin{tabular}{|c|c|}
\hline$\square$ Decrease in Initial Condition (IC) & Increase in Initial Condition (IC \\
\hline IC baseline value: $\mathrm{u}_{\mathrm{T}}(0)=2.857 \times 10^{-3}$ & IC range: $\mathrm{u}_{\mathrm{T}}:\left(2.857 \times 10^{-5}-2.857 \times 10^{-1}\right)$ \\
$\mathrm{u}_{\mathrm{M} 1}(0)=8.93 \times 10^{-4}$ & $\mathrm{u}_{\mathrm{M} 1}:\left(8.93 \times 10^{-6}-8.93 \times 10^{-2}\right)$ \\
$\mathrm{u}_{\mathrm{M} 12}(0)=0.0$ & $\mathrm{u}_{\mathrm{M} 12}:(0.0-0.001)$ \\
$\mathrm{u}_{\mathrm{M} 2}(0)=0.0$ & $\mathrm{u}_{\mathrm{M} 2}:(0.0-0.001)$ \\
\hline
\end{tabular}

Figure 5: Changes in tumour size (see $L_{S}$ in eq. (4)) on days (a) $t=40$ and (b) $t=60$, as we vary the initial conditions (ICs) for each of the four variables from their baseline values (in red colour) to the lower values (cyan colour) and the upper values (black colour) of the indicated IC ranges. Since the baseline values for $u_{M 12}(0)$ and $u_{M 2}(0)$ were zero, we only increased these values to $10^{-3}$.

(panel (b)), as we vary separately each of the rates $\alpha_{m 1}, \alpha_{m 12}, \alpha_{m 21}, \alpha_{m 2}$. The rates $\alpha_{m 1}, \alpha_{m 12}$ are increased/decreased by $90 \%$ from their baseline levels, while rates $\alpha_{m 2}, \alpha_{m 21}$ are increased from their zero baseline levels to 0.01. Panels (c),(d),(e) show the time evolution of tumour $(t \in[0,100])$ as we vary these rates polarisation/re-polarisation rates. As expected, when we decrease $\alpha_{m 12}$ or we increase $\alpha_{m 2}$ we see a decrease in tumour size. However, unexpectedly, a decrease in $\alpha_{m 1}$ (which should reduce the M1 $\rightarrow$ M2 polarisation), seems to cause a significant increase in the tumour population.

In Figure 7 we show the effect of varying at the same time and by the same amount the polarisation rates $\alpha_{m 1}$ and $\alpha_{m 12}$ (where we assume that $\alpha_{m 1}=$ $\alpha_{m 12}=: \alpha_{1}$ ), and the re-polarisation rates $\alpha_{m 2}$ and $\alpha_{m 21}$ (where we assume that $\alpha_{m 2}=\alpha_{m 21}=: \alpha_{2}$ ). Panels (a) and (b) show the magnitude of the changes in tumour size on days $t=40$ and $t=60$, while panels (c) and (d) show the timevariations in $u_{T}(t)$ as we vary simultaneously $\alpha_{m 1}=\alpha_{m 12}$ and $\alpha_{m 2}=\alpha_{m 21}$, respectively.

- Figure 7(c): when we decrease $\alpha_{m 1}=\alpha_{m 12}=\alpha_{1}$ to $\alpha_{1}=0.00001$ we first observe a tumour increase (for $t \in(30,50)$ ) followed by a large decrease (for $t \in(50,80)$ ). The initial tumour increase is unexpected, since a reduction in $\alpha_{1}$ should keep the macrophages in a M1-like phenotype.

- Figure 7(d): when we increase $\alpha_{m 2}=\alpha_{m 21}=\alpha_{2}$, we observe that the tumour can be reduced and eventually eliminated for very large repolarisation rates (e.g., $\left.\alpha_{2}=0.2\right)$. However, in the short time $(t \in(30,50))$ the tumour grows to large sizes; it can even reach its carrying capacity before being killed by the M1-like macrophages. Therefore, these results 
$+90 \%$ of baseline parameter $\square-90 \%$ of baseline parameter $\square$ increased from 0.0 to 0.01
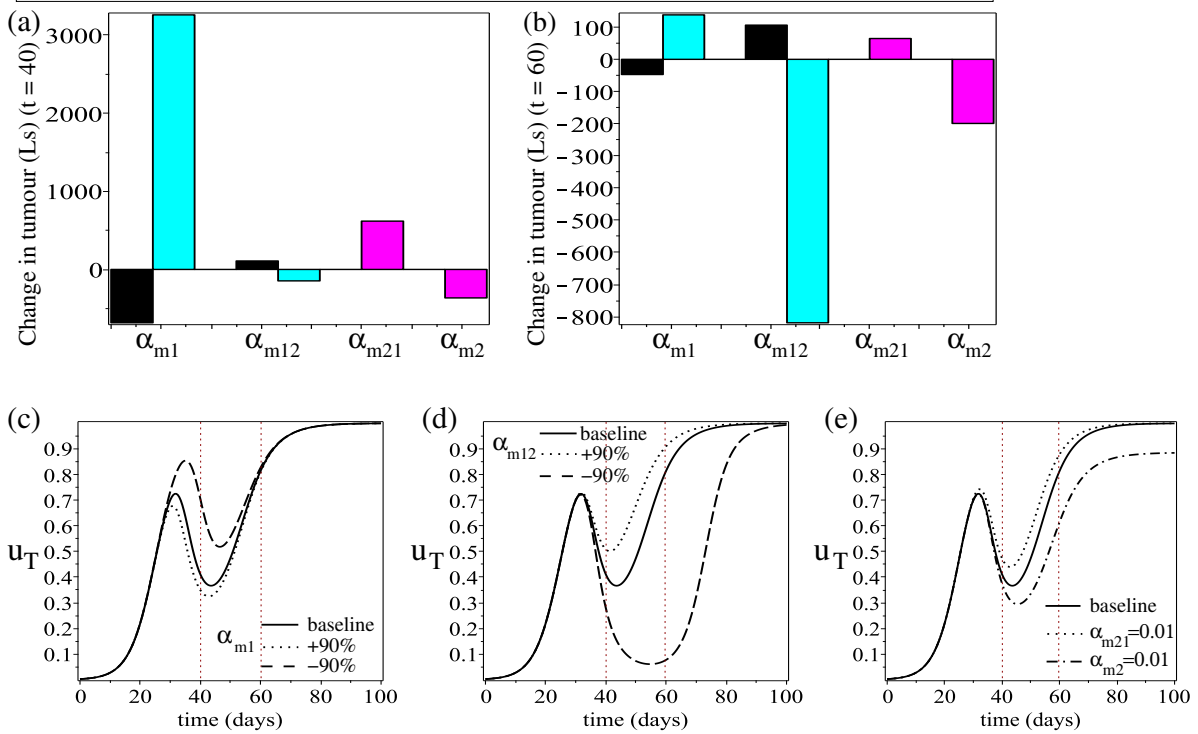

Figure 6: Changes in tumour size (see $L_{s}$ in eq. (4) ) on days (a) $t=40$ and (b) $t=60$, as we vary separately $\alpha_{m 1}, \alpha_{m 12}$ by $\pm 90 \%$ from their baseline values (black and cyan colours), while $\alpha_{m 21}$ and $\alpha_{m 2}$ are increased separately from 0.0 to 0.01 (magenta colour). In (c),(d) we show the time-evolution of the tumour as vary separately by $\pm 90 \%$ (c) $\alpha_{m 1}$ and (d) $\alpha_{m 12}$. In (e) we show the time-evolution of the tumour as we vary separately $\alpha_{m 21}$ and $\alpha_{m 2}$ : each rate is increased from 0.0 (baseline) to 0.01 . The dotted vertical lines indicate the times $t=40$ and $t=60$

suggests that there might be a range for the re-polarisation rates that would lead to optimal treatment. (For murine experiments mice are killed for humane reasons when the tumours grow too large, and thus a potential tumour decay for $t>80$ would not be observed).

To further investigate the unexpected effect of $\alpha_{m 1}$ on tumour growth, in Figure 8 we show the tumour cell volume as we vary: (a) $d_{t 1}$ (to verify whether tumour increase for low $\alpha_{m 1}$ is the result of low M1 phagocytosis); (b) $u_{M 1}(0)$ (to verify whether tumour increase for low $\alpha_{m 1}$ is the result of low initial M1 population); (c) $d_{m 1}$ (to verify whether tumour increase is the result of a continuously low M1 population due to high death rate). It is clear that while an increase in $d_{t 1}$ and $u_{M 1}(0)$ alone, or a decrease in $d_{m 1}$ alone, leads to a temporary reduction in tumour size, when we combine them also with a decrease in $\alpha_{m 1}$ the tumour grows back. A more significant tumour reduction is observed for very low $d_{m 1}$ values (i.e., $d_{m 1} \leq 0.5$ ). Only in this particular case, a decrease in $\alpha_{m 1}$ leads to lower tumour sizes - although the tumour will eventually relapse and grow to its carrying capacity. This temporary decrease in tumour size seems to be the result of higher $\% u_{M 12}$ (see Figure 8(c)(ii)-(ii')).

Since macrophages death rates seem to play an important role in macrophage re-polarising treatments, in Figure 9 we explore the region in the $\left(d_{m 12}, d_{m 1}\right)$ 

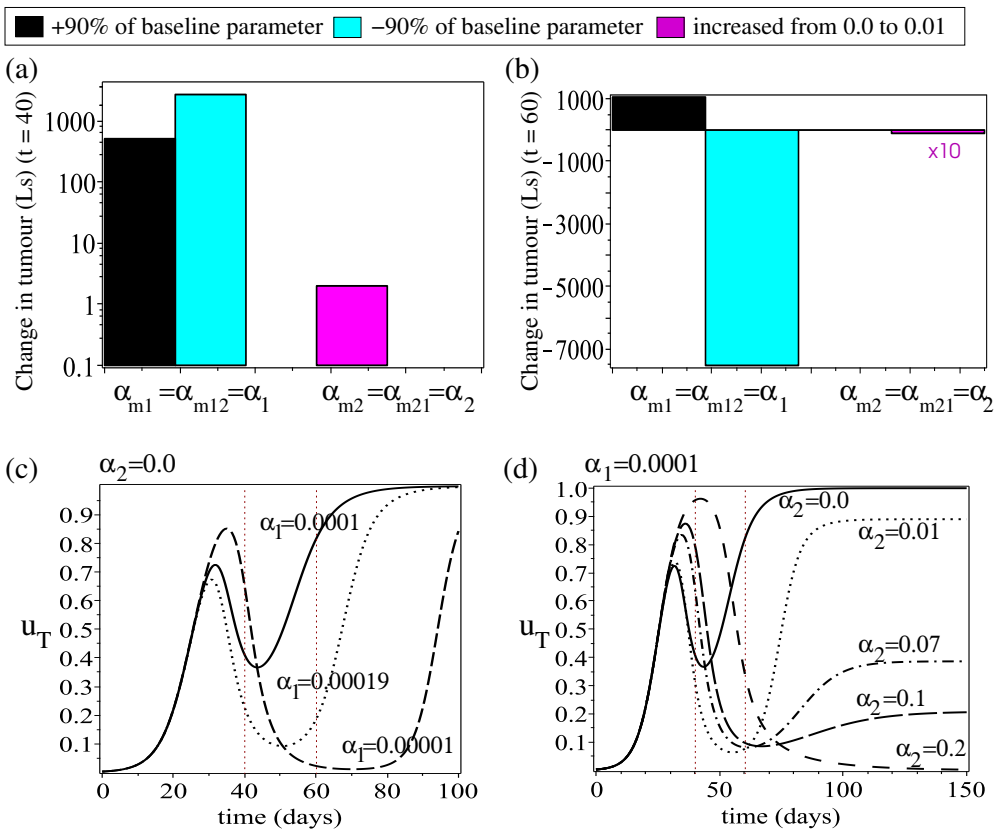

Figure 7: Panels (a),(b) show changes in tumour size (i.e., $L_{S}$ value given by eq. (4)) on days $t=40$ and $t=60$, as we vary at the same time the two polarisation rates $\alpha_{m 1}=\alpha_{m 12}=: \alpha_{1}$ by $\pm 90 \%$ (from a common baseline value of $\alpha_{1}=0.0001$ ) and $\alpha_{m 2}=\alpha_{m 21}=: \alpha_{2}$ (from their baseline value 0.0 to 0.01 ). Since the increase in $\alpha_{2}$ has a very little effect on tumour size on day $t=60$, to improve the visualisation we multiplied the value $L_{S}$ from eq. (4) by a factor of 10. Panel (c) shows the time-evolution of $u_{T}$ when we assume $\alpha_{M 2}=\alpha_{M 21}=0.0$ and we vary $\alpha_{1}:=\alpha_{M 1}=\alpha_{M 12}$. Panel (d) shows the time-evolution of $u_{T}$ when we assume $\alpha_{M 1}=\alpha_{M 12}=0.0001$ and we vary $\alpha_{2}:=\alpha_{M 2}=\alpha_{M 21}$. The dotted vertical lines indicate the times $t=40$ and $t=60$.

space for which a decrease in $\alpha_{m 1}$ (from $10^{3}$ to $10^{4}$ ) leads to a decrease or an increase in tumour size. The thick red curve in the top-left panel corresponds to a stationary tumour (i.e. the dot and dash-dot curves overlap, and the tumour is neither increasing nor decreasing). For three cases corresponding to three corners of this main panel, we show both the time-evolution of the tumour, and the percentage of macrophages corresponding to some of these tumours. We observe that tumour reduction/control for $d_{m 12}=0.14, d_{m 1}=0.45$ is associated with a large percentage of macrophages with mixed M1/M2 phenotypes. In contrast, tumour reduction/control for $d_{m 12}=0.35, d_{m 1}=0.45$ is associated with a very low percentage of macrophages with mixed M1/M2 phenotypes. Hence, the death rates of macrophages with M1-like phenotype or mixed M1/M2-like phenotype seem to play an important role on tumour growth/control/decay, and on the percentage of mixed macrophages during tumour growth/control/decay. We will discuss these results in more detail in Section 4 

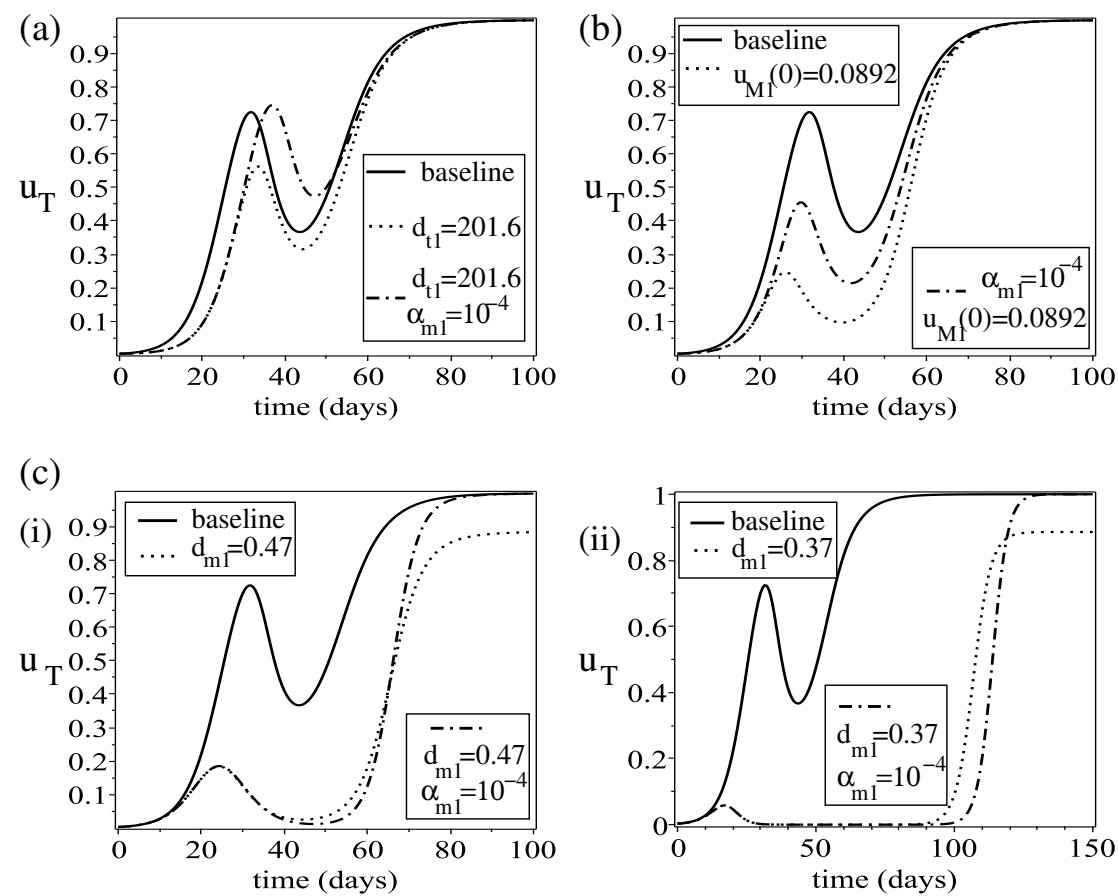

Figure 8: Time-evolution of tumour cell volume as we vary different parameters related to M1 cells. The "baseline" case (continuous curve) corresponds to the parameter values listed in Table 2.1 where $\alpha_{m 1}=10^{-3}$. (a) Vary $d_{t 1}$ alone (dotted curve) or in combination with $\alpha_{m 1}$ (dash-dot curve). To see some difference in tumour dynamics, we had to increase $d_{t 1}$ by a factor of 30: from $d_{t 1}=1.344$ to $d_{t 1}=201.6$ (b) Vary the initial condition $u_{M 1}(0)$ alone (dotted curve) or in combination with $\alpha_{m 1}$ (dash-dot curve). Here we increased the initial condition by a factor of 100: from $u_{M 1}(0)=0.000892$ to $u_{M 1}(0)=0.0892$. (c) Vary $d_{m 1}$ alone (dotted curve) or in combination with $\alpha_{m 1}$ (dash-dot curve). Here we show the effects of medium vs. low values of $d_{m 1}$ : (i) $d_{m 1}=0.6$, (ii) $d_{m 1}=0.5$. For this panel (c) we also show the percentage of various types of macrophages: (i') for the case $d_{m 1}=0.6$, (ii') for the case $d_{m 1}=0.5$.

\subsubsection{Global sensitivity analysis}

To determine the impact that possible interactions between multiple uncertain parameters have on overall tumour dynamics, next we perform a global sensitivity and uncertainty analysis using the classical LHS/PRCC (Latin Hypercube Sampling/Partial Rank Correlation Coefficient) approach [40, 41].

In Figure 10 we show the results of a global sensitivity and uncertainty analysis for: (a) tumour population $\left(u_{T}\right)$, (b) M1 macrophage population $\left(u_{M 1}\right)$, (c) macrophage population with mixed M1/M2 phenotypes $\left(u_{M 12}\right)$, and (d) M2 macrophage population $\left(u_{M 2}\right)$, as we sample independently (100 times) the 14 parameter values within the ranges listed in Table 2.1. Sub-panels (i) show the average population outputs ("mean" and "standard deviations" ( \pm sd) in darker colours, and maximum/minimum values in lighter colours), while sub-panels (ii) 


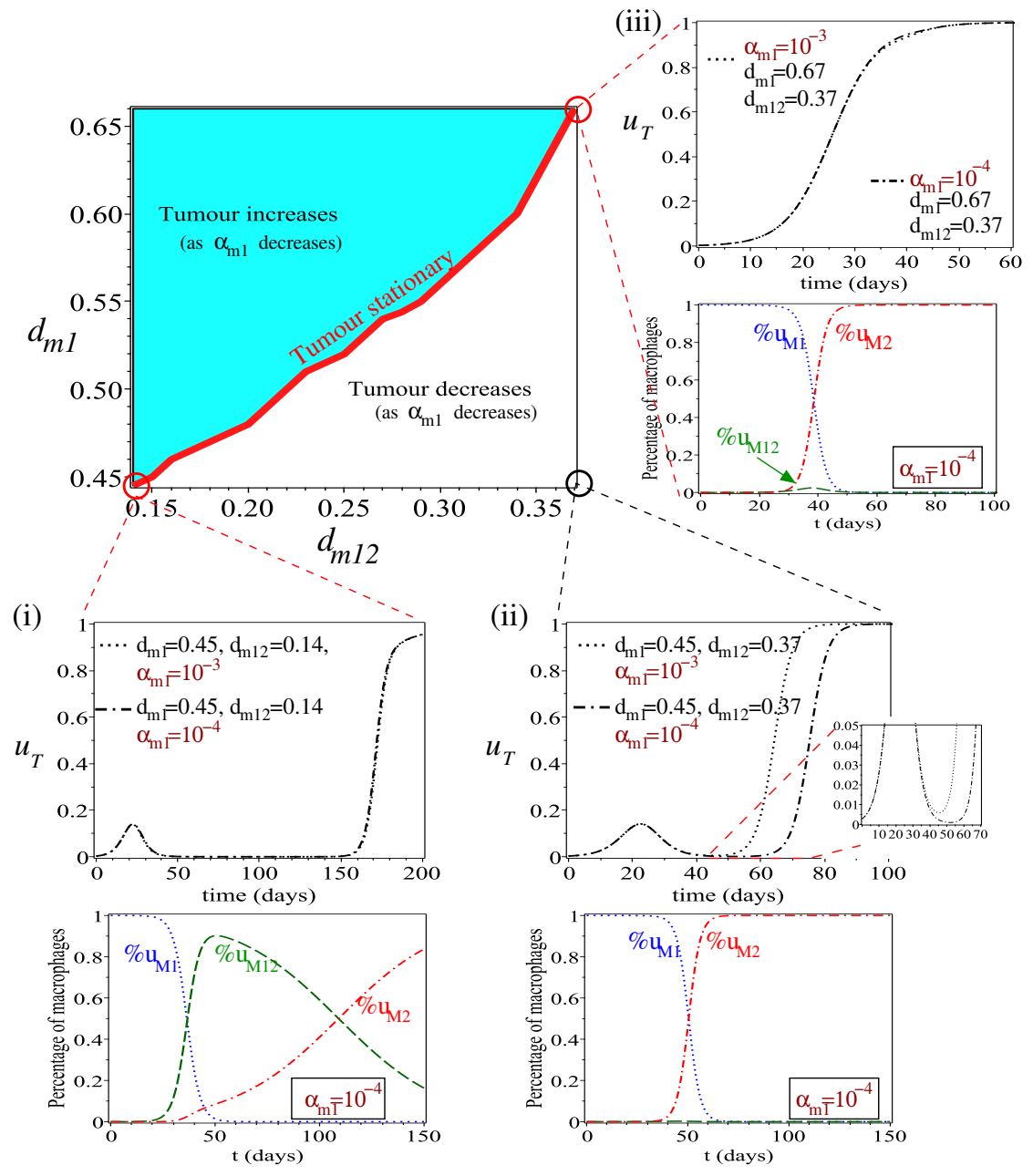

Figure 9: Bifurcation diagram in the $\left(d_{m 12}, d_{m 1}\right)$ parameter space showing the regions where a decrease in $\alpha_{m 1}$ from $10^{-3}$ (dot curves) to $10^{-4}$ (dash-dot curves) leads to a decrease in tumour size (white region) or an increase in tumour size (cyan region). To make it clear what we mean by stationary tumour (i.e. the dot and dash-dot curves overlap), we also show the time-evolution of the tumour and macrophage percentage at three corner points in this parameter space: (i) $d_{m 12}=0.14, d_{m 1}=0.45$; (ii) $d_{m 12}=0.37, d_{m 1}=0.45$; and (iii) $d_{m 12}=0.37, d_{m 1}=0.67$. To clarify that the delay in tumour relapse observed for case (ii) is the result of reduced tumour levels, in this sub-figure we also show a zoom-in of tumour growth curves for $u_{T}<0.05$.

show the PRCC values. For the PRCC analysis, we note that the parameters with large PRCC absolute values are the most important. In particular, values closer to \pm 1 indicate parameters that influence strongly the outcome variable, while the sign indicates the qualitative relation between input parameters and 
output variables (with " + "sign indicating that the parameter is directly proportional to the outcome, and the "-" sign indicating that the parameter is inversely proportional to the outcome). In Figure 10 we see that:

- The parameters with the largest impact on $u_{T}$ are $\alpha_{m 21}, \alpha_{m 2}, d_{t 1}, p_{m 2}$ and $d_{m 12}$. Interestingly, these parameters correspond to the four tumourassociated macrophage targeted therapeutic strategies discussed in 42. antibody-mediated elimination of tumour cells $\left(d_{t 1}\right)$, blockage of monocyte recruitment to tumours $\left(p_{m 2}\right)$, re-polarisation to an M1-like phenotype $\left(\alpha_{m 21}, \alpha_{m 2}\right)$, and suppression of macrophage survival $\left(d_{m 12}\right)$.

- The parameters with the largest impact on $u_{M 1}$ are $\alpha_{m 2}, d_{m 12}$, and $p_{m 2}$.

- The parameter with the largest impact on $u_{M 12}$ is $\alpha_{m 21}$, followed by $\alpha_{m 2}$, $d_{m 12}$ and $p_{m 2}$.

- The parameter with the largest impact on $u_{M 2}$ are $\alpha_{m 2}$, followed by $p_{m 2}$. Among the polarisation rates, the largest impact is provided by $\alpha_{m 12}$.

The above results identified the proliferation/recruitment rate of M2 macrophages $\left(p_{m 2}\right)$ as a parameter important not only for tumour dynamics, but also for the dynamics of all macrophage phenotypes, including $u_{M 1}$ and $u_{M 12}$. Therefore, reducing $p_{m 2}$ will impact tumour growth both directly (through the direct pro-tumour effect of M2 macrophages [43, 26]) and indirectly (through the M1 macrophages and macrophages with mixed M1/M2 phenotypes).

\section{Discussion}

In this study we developed and investigated numerically a new mathematical model for the temporal dynamics between non-small cell lung cancer and macrophages in the lung, with the ultimate goal of shedding some light on the importance of macrophages with mixed phenotypes.

After showing the baseline dynamics of this new model (Figure 4), we started performing a local sensitivity analysis, to gain some understanding of the effects of changes in the initial conditions (Figure 5), as well as changes in those parameters describing macrophage polarisation/re-polarisation rates (Figures 6 - 9). In regard to the initial conditions, our local sensitivity analysis showed that the most important role is played by $u_{M 12}(0)$. For the baseline parameter values investigated in this study (see Table 2.1) we observed that a decrease in the polarisation rate $\alpha_{m 12}$ (i.e., $u_{M 1} \rightarrow u_{M 12}$ polarisation) led to larger tumours, even when we increased the initial level of M1 macrophages $\left(u_{M 1}(0)\right)$ or the M1 macrophages phagocytosis rate $\left(d_{t 1}\right)$. We discovered that this was the result of short half-life of M1 cells (i.e., large $d_{m 1}$; see Figures 8- 9].

Returning to Figure 1(a), one of the main questions of this paper was to shed some light on the role of macrophages with mixed phenotypes on tumour elimination/growth. Through numerical simulations in Figures 8, 9 , we showed that the percentage of $u_{M 12}$ macrophages depends on the elimination rates $d_{1}$ 
(a)

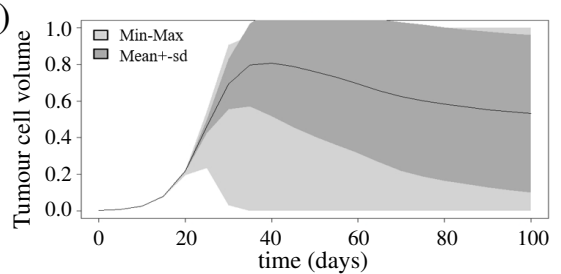

(c)

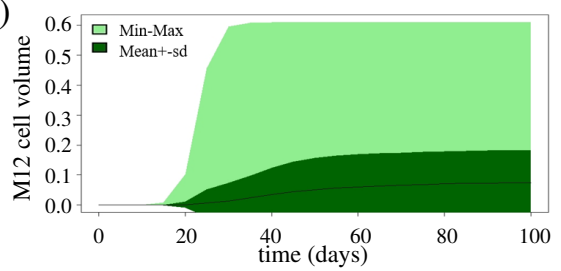

(b)

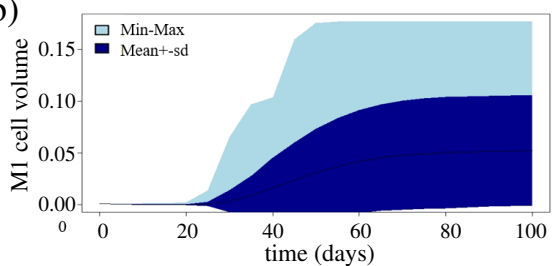

(d)

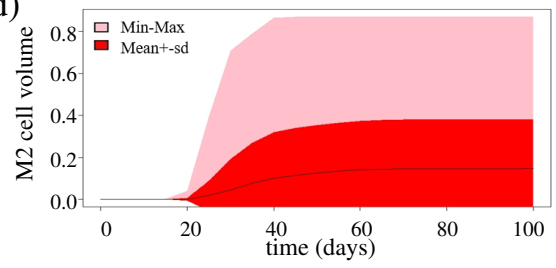

(e)

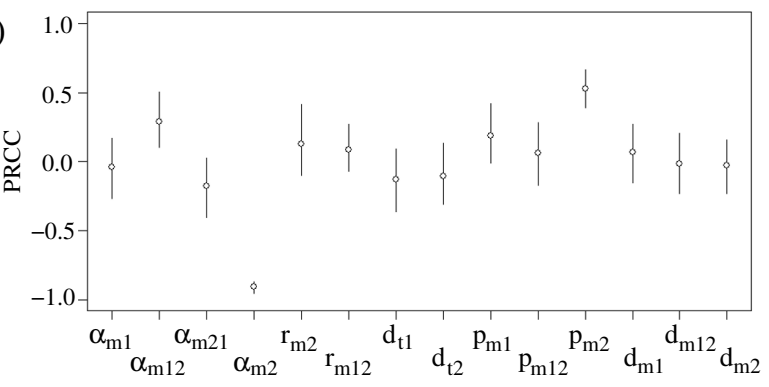

Figure 10: Global sensitivity and uncertainty analysis for variables (a) $u_{T}$, (b) $u_{M 1}$, (c) $u_{M 12}$, (d) $u_{M 2}$ as we vary 14 parameters within the ranges specified in Table 2.1 Sub-panels (i) show the mean+standard deviation, together with $\max / \min$ values of these variables as we vary $t$. Sub-panels (ii) show the PRCC values corresponding to each of the parameters varied in Table 2.1

and $d_{12}$ (and it probably on other parameters as well). In particular, tumour decay/control can occur for both low and high $u_{M 12}$ percentages. Therefore, our theoretical study suggests that unless we know exactly the elimination rates of macrophages with different phenotypes (M1 or mixed M1/M2) we cannot use the macrophages with mixed phenotypes as predictors of tumour elimination (and patient survival).

The results presented in this study depend heavily on the parameters used for the simulations. Some of these parameter values were obtained from in-vitro and ex-vivo experiments 30,33 , and therefore they could be different from the in-vivo murine parameters and even more from the in-vivo human parameters. Unfortunately, we do not have in-vivo data to parametrise these mathematical models, and our best approach was a sensitivity and uncertainty analysis to understand the extent of variations in model outcomes. Global sensitivity 
analysis (Figure 10 revealed the parameters with the largest impact for tumour dynamics $\left(\alpha_{m 21}, \alpha_{m 2}, d_{t 1}, p_{m 2}\right.$ and $\left.d_{m 12}\right)$, and interestingly these parameters were also the parameters involved in four of the macrophage-targeted treatment approaches for cancer as identified in [42]: re-polarisation of macrophages towards an M1-like phenotype $\left(\alpha_{m 21}, \alpha_{m 2}\right)$, suppression of tumour-associated macrophages survival $\left(d_{m 12}\right)$, blockade of macrophage recruitment $\left(p_{m 2}\right)$, and antibody-mediated elimination of tumour cells by macrophages $\left(d_{t 1}\right)$.

Overall, the results of this study emphasise the need for a better experimental understanding of the kinetics (doubling time, half lives) of macrophages with different phenotypes that can be found inside solid tumours (especially the macrophages with mixed phenotypes). Most of the experimental studies in the literature focus on the kinetics of $\mathrm{T}$ cells [44, 45, but given the importance of tumour-associated macrophages on tumour evolution, more experimental studies are necessary to better understand the macrophage kinetics. Unfortunately, the lack of robust macrophage markers can lead to inaccurate macrophage counts [16, which further impacts our hope of reliable data on macrophage kinetics. Until more data will become available, we have to continue using modelling and computational approaches to propose hypotheses regarding the macrophage dynamics and their interactions with various components of the tumour microenvironment. 


\section{Appendix A. Spatially-homogeneous steady states}

We have seen in Figures $7(\mathrm{~d})$ and 8 (c),(d) that changes in some parameters can lead to lower tumour sizes in the long term (for $\alpha_{m 21}, \alpha_{m 2}>0$ ), in contrast to the case $\alpha$. To understand better this long-term dynamics of model (1), in the following we summarise the steady states exhibited by this model by focusing on two case: (i) the baseline case characterised by $\alpha_{m 2}=\alpha_{m 21}=0$, and (ii) the treatment case characterised by $\alpha_{m 2}, \alpha_{m 21}>0$.

Proposition 1. For the baseline case (i) with $\alpha_{m 2}, \alpha_{m 21}=0$, model (1) can exhibit the following steady states:

- Tumour-Free Immune-Free (TFIF) state: $\left(u_{T}^{*}, u_{M 1}^{*}, u_{M 12}^{*}, u_{M 2}^{*}\right)=(0,0,0,0)$, which exists for all parameter values;

- Tumour-Free M2-Present (TFM2P) steady state: $\left(u_{T}^{*}, u_{M 1}^{*}, u_{M 12}^{*}, u_{M 2}^{*}\right)=$ $\left(0,0,0, \frac{K_{m}\left(p_{m 2}-d_{m 2}\right)}{p_{m 2}}\right)$, which exists for $p_{m 2}>d_{m 2}$;

- Tumour-Free M12-Present (TFM12P) steady state: $\left(u_{T}^{*}, u_{M 1}^{*}, u_{M 12}^{*}, u_{M 2}^{*}\right)$ $=\left(0,0, \frac{K_{m}\left(p_{m 12}-d_{m 12}\right)}{p_{m 12}}, 0\right)$, which exists for $p_{m 12}>d_{m 12}$;

- Tumour-Free M1-Present (TFM1P) steady state: $\left(u_{T}^{*}, u_{M 1}^{*}, u_{M 12}^{*}, u_{M 2}^{*}\right)=$ $\left(0, \frac{K_{m}\left(p_{m 1}-d_{m 1}\right)}{p_{m 1}}, 0,0\right)$, which exists only for $p_{m 1}>d_{m 1}$, an inequality not satisfied by the baseline parameter values in Table 2.1:

- Tumour-Present Immune-Free (TPIF) steady state: $\left(u_{T}^{*}, u_{M 1}^{*}, u_{M 12}^{*}, u_{M 2}^{*}\right)$ $=\left(K_{t}, 0,0,0\right)$, which exits for all parameter values;

- Tumour-Present M2-Present (TPM2P) steady state: $\left(u_{T}^{*}, u_{M 1}^{*}, u_{M 12}^{*}, u_{M 2}^{*}\right)$ $=\left(K_{t}, 0,0, \frac{p_{m 2}-d_{m 2}}{p_{m 2}} K_{m}\right)$ which exists for $p_{m 2}>d_{m 2}$;

- Tumour-Present M12-M2-Present (TPM12M2P) steady state: $\left(u_{T}^{*}, u_{M 1}^{*}, u_{M 12}^{*}, u_{M 2}^{*}\right)$ $=\left(u_{T}^{*}, 0, u_{M 12}^{*}, u_{M 2}^{*}\right)$ with the non-zero states connected via the following equations:

$$
\begin{aligned}
u_{M 12}^{*} & =\frac{K_{m} \frac{\left[p_{m 12}\left(u_{T}^{*}+K_{t}^{*}\right)-d_{m 12}\left(u_{T}^{*}+K_{t}^{*}\right)+\alpha_{m 12} u_{T}^{*}\right]}{p_{m 12}\left(u_{T}+K_{t}^{*}\right)}}{\alpha_{m 12} u_{T}^{*}}+1 \\
u_{M 2}^{*} & =K_{m}\left(1-\frac{d_{m 12}}{p_{m 12}}+\frac{\alpha_{m 12}}{p_{m 12}} \frac{u_{T}^{*}}{\left.u_{T}^{*}+K_{t}^{*}\right) p_{m 2}\left[\frac{d_{m 12}}{p_{m 12}}-\frac{\alpha_{m 12}}{p_{m 12}} \frac{u_{T}^{*}}{u_{T}^{*}+K_{t}^{*}}\right]-d_{m 2}\left(u_{T}^{*}+K_{t}^{*}\right)}-u_{M 12}^{*}\right. \\
0 & =p_{t}\left(1-\frac{u_{T}^{*}}{K_{t}}\right)\left(1+r_{m 2} u_{M 2}^{*}+r_{m 12} u_{M 12}^{*}\right)-d_{t 2} u_{M 12}^{*} \cdot
\end{aligned}
$$

- Tumour-Present Immune-Present (TPIP) steady state: $\left(u_{T}^{*}, u_{M 1}^{*}, u_{M 12}^{*}, u_{M 2}^{*}\right)$.

Proposition 2. For the case (ii) when a macrophage re-polarisation treatment is considered (i.e., $\alpha_{m 2}, \alpha_{m 21}>0$ ), model (1) can exhibit the following tumourfree and tumour-present steady states: 
- Tumour-Free Immune-Free (TFIF) state: $\left(u_{T}^{*}, u_{M 1}^{*}, u_{M 12}^{*}, u_{M 2}^{*}\right)=(0,0,0,0)$, which exists for all parameter values;

- Tumour-Free M1-cells Present (TFM1P) state: $\left(u_{T}^{*}, u_{M 1}^{*}, u_{M 12}^{*}, u_{M 2}^{*}\right)=$ $\left(0, K_{m} \frac{p_{m 1}-d_{m 1}}{p_{m 1}}, 0,0\right)$, which exists only if $p_{m 1}>d_{m 1}$.

- Tumour-Free M2-cells Free (TFM2F) state: $\left(u_{T}^{*}, u_{M 1}^{*}, u_{M 12}^{*}, u_{M 2}^{*}\right)=\left(0, u_{M 1}^{*}, u_{M 12}^{*}, 0\right)$, with

$$
\begin{aligned}
u_{M 1}^{*} & =\frac{\alpha_{m 21} K_{m}\left(\alpha_{m 21}+d_{m 12}-p_{m 12}\right)}{\left(p_{m 1}-p_{m 12}\right) \alpha_{m 21}-d_{m 1} p_{m 12}+d_{m 12} p_{m 1}} \\
u_{M 12}^{*} & =\frac{K_{m}\left(d_{m 12}+\alpha_{m 21}-p_{m 12}\right)\left(-d_{m 1} p_{m 12}+p_{m 1}\left(d_{m 12}+\alpha_{m 21}\right)\right)}{p_{m 12}\left(p_{m 12}\left(d_{m 1}+\alpha_{m 21}\right)-p_{m 1}\left(d_{m 12}+\alpha_{m 21}\right)\right)} .
\end{aligned}
$$

- Tumour-Free, Immune response Present (TFIP) state: $\left(u_{T}^{*}, u_{M 1}^{*}, u_{M 12}^{*}, u_{M 2}^{*}\right)$ $=\left(0, u_{M 1}^{*}, u_{M 12}^{*}, u_{M 2}^{*}\right)$, with

$$
u_{M 1}^{*}=\frac{\alpha_{m 2} \alpha_{m 21} K_{m} p_{m 2}\left(d_{m 2}+\alpha_{m 2}-p_{m 2}\right)}{\left(\left(-d_{m 1}-\alpha_{m 21}\right) \alpha_{m 2}-d_{m 1}\left(\alpha_{m 21}+d_{m 12}\right)\right) p_{m 2}^{2}-p_{m 1} p_{m 12}\left(d_{m 2}+\alpha_{m 2}\right)^{2}},
$$

$$
\begin{array}{r}
u_{M 12}^{*}=\frac{\alpha_{m 2}\left(-d_{m 1} p_{m 2}+p_{m 1}\left(\alpha_{m 2}+d_{m 2}\right)\right)\left(\alpha_{m 2}+d_{m 2}-p_{m 2}\right) K_{m}}{\left(\left(-d_{m 1}-\alpha_{m 21}\right) \alpha_{m 2}-d_{m 1}\left(\alpha_{m 21}+d_{m 12}\right)\right) p_{m 2}^{2}-p_{m 1} p_{m 12}\left(\alpha_{m 2}+d_{m 2}\right)^{2}} \\
+\left(\alpha+m 2 p_{m 1}+\left(\alpha_{m 21}+d_{m 12}\right) p_{m 1}+d_{m 1} p_{m 12}\right)\left(\alpha_{m 2}+d_{m 2}\right) p_{m 2}
\end{array}
$$

and

$$
\begin{aligned}
& {\left[p_{m 1}\left(\alpha_{m 2}+d_{m 2}\right)-d_{m 1} p_{m 2}\right]\left(\alpha_{m 2}+d_{m 2}-p_{m 2}\right)} \\
& u_{M 2}^{*}=\frac{\cdot\left(\left(-\alpha_{m 21}-d_{m 12}\right) p_{m 2}+p_{m 12}\left(\alpha_{m 2}+d_{m 2}\right)\right) K_{m}}{p_{m 2}\left(\left(\left(-d_{m 1}-\alpha_{m 21}\right) \alpha_{m 2}-d_{m 1}\left(\alpha_{m 21}+d_{m 12}\right)\right) p_{m 2}^{2}-p_{m 1} p_{m 12}\left(\alpha_{m 2}+d_{m 2}\right)^{2}\right)} \text {. } \\
& +p_{m 2}\left(\left(\left(\alpha_{m 2}+\alpha_{m 21}+d_{m 12}\right) p_{m 1}+d_{m 1} p_{m 12}\right)\left(\alpha_{m 2}+d_{m 2}\right) p_{m 2}\right)
\end{aligned}
$$

- Tumour-Present Immune-Free (TPIF) state: $\left(u_{T}^{*}, u_{M 1}^{*}, u_{M 12}^{*}, u_{M 2}^{*}\right)=\left(K_{t}, 0,0,0\right)$, which exits for all parameter values;

- Tumour-Present M1, M12 and M2 Immune response Present (TPIP) state: $\left(u_{T}^{*}, u_{M 1}^{*}, u_{M 12}^{*}, u_{M 2}^{*}\right)=\left(u_{T}^{*}, u_{M 1}^{*}, u_{M 12}^{*}, u_{M 2}^{*}\right)$.

Remark 3. Both cases investigated above, namely (i) no re-polarising treatment $\left(\alpha_{m 2}=\alpha_{m 21}=0\right)$ and (ii) re-polarising treatment $\left(\alpha_{m 2}, \alpha_{m 21}>0\right)$ had a tumour-only steady state (TPIF) and coexistence steady states (TPIP). However, case (i) can exhibit two other steady states characterised by the presence of tumour cells and the absence of M1-like cells $\left(u_{M 1}^{*}=0\right)$ : the TPM2P and TPM12M2P states. This suggests that in the absence of any external treatment to re-polarise the macrophages towards an M1-like phenotype, the tumours are always formed of M2-like macrophages or macrophages with a mixed M1/M2 phenotype. 
Stability of steady states. The linear stability of the above steady states is controlled by the eigenvalues of the Jacobian matrix associated with the system (1):

$$
\begin{aligned}
& J\left(u_{T}^{*}, u_{M 1}^{*}, u_{M 12}^{*}, u_{M 2}^{*}\right)=\left(\begin{array}{llll}
a_{11} & a_{12} & a_{13} & a_{14} \\
a_{21} & a_{22} & a_{23} & a_{24} \\
a_{31} & a_{32} & a_{33} & a_{34} \\
a_{41} & a_{42} & a_{43} & a_{44}
\end{array}\right), \text { with } \\
a_{11}= & p_{t}\left(1-\frac{2 u_{T}^{*}}{K_{t}}\right)\left(r_{m 12} u_{M 12}^{*}+r_{m 2} u_{M 2}^{*}+1\right)-d_{t 1} u_{M 1}^{*}-d_{t 2} u_{M 12}^{*}, \\
a_{12}= & -d_{t 1} u_{T}^{*}, \\
a_{13}= & p_{t} u_{T}^{*}\left(1-\frac{u_{T}^{*}}{K_{t}}\right) r_{m 12}-d_{t 2} u_{T}^{*}, \\
a_{14}= & p_{t} u_{T}^{*}\left(1-\frac{u_{T}^{*}}{K_{t}}\right) r_{m 2}, \\
a_{21}= & \frac{\alpha_{m 1} u_{M 1}^{*}}{u_{T}^{*} K_{t}^{*}}\left(\frac{u_{T}^{*}}{u_{T}^{*}+K_{t}^{*}}-1\right), \\
a_{22}= & p_{m 1}\left(1-\frac{u_{M 1}^{*}+u_{M 12}^{*}+u_{M 2}^{*}}{K_{m}}\right)-\frac{p_{m 1}}{u_{M 1}^{*}}-d_{m 1}-\frac{\alpha_{m 1} u_{T}^{*}}{u_{T}^{*}+K_{t}^{*}}, \\
a_{23}= & -\frac{p_{m 1} u_{M 1}^{*}}{K_{m}}+\alpha_{m 21}, \\
a_{24}= & -\frac{p_{m 1} u_{M 1}^{*}}{K_{m}}, \\
a_{31}= & \frac{\alpha_{m 1} u_{M 1}^{*}}{u_{T}^{*}+K_{t}^{*}}\left(1-\frac{u_{M 1}^{*}}{u_{T}^{*}+K_{t}^{*}}\right)+\frac{\alpha_{m 12} u_{M 12}^{*}}{u_{T}^{*}+K_{t}^{*}}\left(\frac{u_{M 12}^{*}}{u_{T}^{*}+K_{t}^{*}}-1\right), \\
a_{32}= & \frac{\alpha_{m 1} u_{T}^{*}}{u_{T}^{*}+K_{t}^{*}}-\frac{p_{m 12} u_{M 12}^{*}}{K_{m}}, \\
a_{33}= & p_{m 12}\left(1-\frac{u_{M 1}^{*}+2 u_{M 12}^{*}+u_{M 2}^{*}}{K_{m}}\right)-d_{m 12}-\alpha_{m 21}-\frac{\alpha_{m 12} u_{T}^{*}}{u_{T}^{*}+K_{t}^{*}}, \\
a_{34}= & \alpha_{m 2}-\frac{p_{m 12} u_{M 12}^{*}}{K_{m}}, \\
a_{41}= & \frac{\alpha_{m 12} u_{M 12}^{*}}{u_{T}^{*}+K_{t}^{*}}\left(1-\frac{u_{T}^{*}}{u_{T}^{*}+K_{t}^{*}}\right), \\
a_{42}= & -\frac{p_{m 2} u_{M 2}^{*}}{K_{m}}, \\
a_{43}= & \frac{\alpha_{m 12} u_{T}^{*}}{u_{T}^{*}+K_{t}^{*}}-\frac{p_{m 2} u_{M 2}^{*}}{K_{m}}, \\
a_{44} & p_{m 2}\left(1-\frac{u_{M 1}^{*}+u_{M 12}^{*}+u_{M 2}^{*}}{K_{m}}\right)-\frac{p_{m 2} u_{M 2}^{*}}{K_{m}}-d_{m 2}-\alpha_{m 2} . \\
a_{m} &
\end{aligned}
$$

Proposition 3. The steady states exhibited by model (1) for the baseline case $\alpha_{m 2}=\alpha_{m 21}=0$ have the following linear stability: 
- The TFIF state is always unstable.

- The TFM2P state is always unstable when it exists (i.e., for $p_{m 2}>d_{m 2}$ ).

- The TFM12P state is stable provided that $\frac{d_{m 12}}{p_{m 12}}<\min \left\{\frac{d_{m 2}}{p_{m 2}}, \frac{d_{m 1}}{p_{m 1}}\right\}$, and $p_{t} r_{m 12}<d_{t 2}$.

- The TFM1P state is stable provided that $\frac{d_{m 1}}{p_{m 1}}<\min \left\{\frac{d_{m 2}}{p_{m 2}}, \frac{d_{m 12}}{p_{m 12}}\right\}$ and $p_{t} p_{m 1}<d_{t 1} K_{m}\left(p_{m 1}-d_{m 1}\right)$.

- The TPIF state is stable provided that $p_{m 2}<d_{m 2}$ and $p_{m 1}<d_{m 1}$ and $p_{m 12}<d_{m 12}$.

- The TPM2P state is stable provided that $\frac{d_{m 2}}{p_{m 2}}<\min \left\{\frac{d_{m 1}}{p_{m 1}}, \frac{d_{m 12}}{p_{m 12}}\right\}$.

The stability of TPM12M2P and TPIP states (for the case $\alpha_{m 2}=\alpha_{m 21}=0$ ) is more difficult to investigate for general parameters. Also the stability of the steady states corresponding to the case $\alpha_{m 2}, \alpha_{m 21}>0$ is difficult to investigate for the general parameters (the exceptions being the TFIF and TFM1P states, whose stability is given by the same conditions as in Proposition 3). For these reasons, the following Remark summarises the stability of all steady states for the baseline parameter values in Table 2.1 .

Remark 4. For the baseline parameter values given in Table 2.1 (with $\alpha_{m 2}=$ $\left.\alpha_{m 21}=0\right)$, the steady states TFM1P, TPM12M2P and TPIP do not exist.

The stability of the existent steady states is as follows: the TFIF state is unstable (saddle), the TFM2P state is unstable (saddle), the TFM12P state is unstable (saddle), the TPIF state is unstable (saddle), and the TPM2P state is stable (node). These results explain the dynamics observed in Figure 4, where the solutions approach the only stable steady state: the TPM2P state.

For the case $\alpha_{m 2}=\alpha_{m 21}=0.1$ (and the rest of parameters as in Table 2.1), the steady state TFM1P does not exist.

The stability of the existent steady states is as follows: the TFIF state is unstable (saddle), the TFM2F state is unstable (saddle), the TFIP state is unstable (saddle), the TPIF state is unstable (saddle), and TPIP is stable (node). This explains the dynamics observed in Figure $7(d)$, where the solutions approach the only stable steady state: the TPIP state.

[1] D. Carbone, D. Gandara, S. Antonia, C. Zielinski, L. Paz-Ares, Non-small cell lung cancer: role of the immune system and potential for immunotherapy, J. Thorac. Oncol. 10 (7) (2015) 974-984.

[2] C. Gridelli, A. Rossi, D. Carbone, J. Guarize, N. Karachaliou, T. Mok, F. Petrella, L. Spaggiari, R. Rosell, Non-small-cell lung cancer, Nature Reviews 1 (2015) 15009.

[3] B. Stankovic, H. Bjøhovde, R. Skarshaug, H. Aamodt, A. Frafjord, E. Müller, C. Hammarström, K. Beraki, E. Baekkevold, P. Woldbaek, 
A. Helland, O. Brustugun, I. Øynebråten, A. Corthay, Immune cell composition in human non-small cell lung cancer, Frontiers in Immunology 3 (2019) 3101.

[4] J. Kargl, S. Busch, G. Yang, K.-H. Kim, M. Hanke, H. Metz, J. Hubbard, S. Lee, D. Madtes, M.W.McIntosh, A. M. Houghton, Neutrophils dominate the immune cell composition in non-small cell lung cancer, Nat. Commun. 8 (2017) 14381.

[5] F. Dai, L. Liu, G. Che, N. Yu, Q. Pu, S. Zhang, J. Ma, L. Ma, Z. You, The number and microlocalisation of tumour-associated immune cells are associated with patient's survival time in non-small cell lung cancer, BMC Cancer 10 (2010) 220.

[6] M. Tamminga, T. Hiltermann, E. Schuuring, W. Timens, R. Fehrmann, Immune microenvironment composition in non-small cell lung cancer and its association with survival, Clinical \& Translational Immunology 9 (2020) e1142.

[7] A. Mantovani, S. Sozzani, M. Locati, P. Allavena, A. Sica, Macrophage polarization: tumor-associated macrophages as a paradigm for polarized m2 mononuclear phagocytes, Trends in Immunology 23 (11) (2002) 549555 .

[8] J. Ma, L. Liu, G. Che, N. Yu, F. Dai, Z. You, The $\mathrm{m} 1$ form of tumorassociated macrophages in non-small cell lung cancer is positively associated with survival time, BMC cancer 10 (1) (2010) 112.

[9] C. Ohri, A. Shikotra, R. Green, D. Waller, P. Bradding, Macrophages within nsclc tumour islets are predominantly of a cytotoxic $\mathrm{m} 1$ phenotype associated with extended survival, European Respiratory Journal 33 (1) (2009) 118-126.

[10] L. Cao, X. Che, X. Qiu, Z. Li, B. Yang, S. Wang, K. Hou, Y. Fan, X. Qu, Y. Liu, M2 macrophage infiltration into tumor islets leads to poor prognosis in non-small-cell lung cancer, Cancer Management and Research 11 (2019) 6125 .

[11] J. Jackute, M. Zemaitis, D. Pranys, B. Sitkauskiene, S. Miliauskas, $\mathrm{S}$. Vaitkiene, R. Sakalauskas, Distribution of $\mathrm{m} 1$ and $\mathrm{m} 2$ macrophages in tumor islets and stroma in relation to prognosis of non-small cell lung cancer, BMC Immunology 19 (1) (2018) 3.

[12] M. Rakaee, L.-T. R. Busund, S. Jamaly, E.-E. Paulsen, E. Richardsen, S. Andersen, S. Al-Saad, R. Bremnes, T. Donnem, T. Kilvaer, Prognostic value of macrophage phenotypes in resectable non-small cell lung cancer assessed by multiplex immunohistochemistry, Neoplasia 21 (3) (2019) 282293. 
[13] Z. Xu, Y. Gu, C.-Z. Wang, Y. Jin, X. Wen, J.-C. Ma, L. Tang, Z. Mao, J. Qian, J. Lin, The M2 macrophage marker CD206: a novel prognostic indicator for acute myeloid leukemia, Oncoimmunology 9 (1) (2020) e1683347.

[14] S. Singhal, J. Stadanlick, M. Annunziata, A. Rao, P. Bhojnagarwala, S. O'Brien, E. Moon, E. Cantu, G. Danet-Desnoyers, H.-J. Ra, L. Litzky, T. Akimova, U. Beier, W. Hancock, S. Albelda, E. Eruslanov, Human tumor-associated monocytes/macrophages and their regulation of $\mathrm{T}$ cell responses in early-stage lung cancer, Sci. Transl. Med. 11 (2019) 479.

[15] J. Quatromoni, E. Eruslanov, Tumour-associated macrophages: function, phenotype and link to prognosis in human lung cancer, Am. J. Transl. Res. 4 (4) (2012) 376-389.

[16] S. Jayasingam, M. Citartan, T. Thang, A. Zin, E. Ch'ng, Evaluating the polarisation of tumour-associated macrophages into M1 and M2, Frontiers in Oncology 9 (2020) 1512.

[17] N. Almuallem, D. Trucu, R. Eftimie, Oncolytic viral therapies and the delicate balance between virus-macrophage-tumour interactions: A mathematical approach, Math. Biosci. Eng. 18 (1) (2020) 764-799.

[18] N. Y. den Breems, R. Eftimie, The re-polarisation of $\mathrm{m} 2$ and $\mathrm{m} 1$ macrophages and its role on cancer outcomes, Journal of Theoretical Biology 390 (2016) 23-39.

[19] R. Eftimie, H. Hamam, Modelling and investigation of the CD4 ${ }^{+} \mathrm{T}$ cells macrophages paradox in melanoma immunotherapies, J. Theor. Biol. 420 (2017) 82-104.

[20] R. Eftimie, Investigation into the role of macrophages heterogeneity on solid tumour aggregations, Mathematical Biosciences 322 (2020) 108325.

[21] X. Li, M. Jolly, J. George, K. Pienta, H. Levine, Computational modeling of the crosstalk between macrophage polarization and tumour cell plasticity in the tumour microenvironment, Front. Oncol. 9 (2019) 10.

[22] F. Leonard, L. T. Curtis, M. J. Ware, T. Nosrat, X. Liu, K. Yokoi, H. B. Frieboes, B. Godin, Macrophage polarization contributes to the antitumoral efficacy of mesoporous nanovectors loaded with albumin-bound paclitaxel, Frontiers in immunology 8 (2017) 693.

[23] F. Leonard, L. Curtis, A. Hamed, C. Zhang, E. Chau, D. Sieving, B. Godin, H. Frieboes, Nonlinear response to cancer nanotherapy due to macrophage interactions revealed by mathematical modeling and evaluated in a murine model via CRISPR-modulated macrophage polarisation, Cancer Immunol. Immunother. 69 (5) (2020) 731-744. 
[24] Y. Louzoun, C. Xue, G. Lesinski, A. Friedman, A mathematical model for pancreatic cancer growth and treatments, J. Theor. Biol. 351 (2014) 74-82.

[25] S. Benzekry, C. Lamont, A. Beheshti, A. Tracz, J. Ebos, L. Hlatky, P. Hahnfeldt, Classical mathematical models for description and prediction of experimental tuour growth, PLoS Comput. Biol. 10 (8) (2014) e1003800.

[26] J. Chen, W. Sun, H. Zhang, J. Ma, P. Xu, Y. Yu, H. Fang, L. Zhou, J. Lv, J. Xie, Y. Liu, K. Tang, B. Huang, Macrophages reprogrammed by lung cancer microparticles promote tumour development via release of IL- $1 \beta$, Cell. Mol. Immunol. (2019) 1-12.

[27] A. A. Patel, Y. Zhang, J. N. Fullerton, L. Boelen, A. Rongvaux, A. A. Maini, V. Bigley, R. A. Flavell, D. W. Gilroy, B. Asquith, et al., The fate and lifespan of human monocyte subsets in steady state and systemic inflammation, Journal of Experimental Medicine 214 (7) (2017) 1913-1923.

[28] E. Soucie, Z. Weng, L. Geirsdøttir, K. Molawi, J. Maurizio, R. Fenouil, N. Mossadegh-Keller, G. Gimenez, L. VanHille, M. Beniazza, J. favret, C. berruyer, P. Perrin, N. Hacohen, J. Andrau, P. Ferrier, P. Dubreuil, A. Sidow, M. Sieweke, Lineage-specific enhancers activate self-renewal genes in macrophages and embryonic stem cells, Science 351 (6274) (2016) $\operatorname{aad} 5510$.

[29] A. Poh, M. Ernst, Targeting macrophages in cancer: from bench to bedside, Frontiers in Oncology 8 (2018) 49.

[30] V. Chitu, Y.-G. Yeung, W. Yu, S. Nandl, E. Stanley, Measurement of macrophage growth and differentiation, Curr. Protoc. Immunol. (2011) 126.

[31] P. Italiani, D. Boraschi, From monocytes to M1/M2 macrophages: phenotypical vs. functional differentiation, Frontiers in Immunology 5 (2014) $47-68$.

[32] M. Heusinkveld, S. van der Burg, Identification and manipulation of tumour associated macrophages in human cancers, Journal of Translational Medicine 9 (2011) 216.

[33] T. Sato, M. Morita, R. Tanaka, Y. Inoue, M. Nomura, Y. Sakamoto, K. Miura, S. Ito, I. Sato, N. Tanaka, J. Abe, S. Takahashi, M. Kawai, M. Sato, Y. Hippo, H. Shima, Y. Okada, N. Tanuma, Ex vivo model of non-small cell lung cancer using mouse lung epithelial cells, Oncology Letters 14 (2017) 6863-6868.

[34] H. Yamaji, T. Iizasa, E. Koh, M. Suzuki, M. Otsuji, H. Chang, S. Motohashi, S. Yokoi, K. Hiroshima, M. Tagawa, T. Nakayama, T. Fujisawa, Correlation between interleukin 6 production and tumour proliferation in non-small cell lung cancer, Cancer Immunol. Immunother. 53 (786-792). 
[35] P. Haley, B. Muggenburg, D. Weissman, D. Bice, Comparative morphology and morphometry of alveolar macrophages from size mammalian species, American Journal of Anatomy 4 (1991) 401-407.

[36] U. D. Monte, Does the cell number $10^{9}$ still really fit one gram of tumour tissue?, Cell Cycle 8 (3) (2009) 505-506.

[37] E. Redente, D. Orlicky, R. Bouchard, A. Malkinson, Tumour signalling to the bone marrow changes the phenotype of monocytes and pulmonary macrophages during urethane-induced primary lung tumorigenesis in $a / j$ mice, Am. J. Pathol. 170 (2007) 693-708.

[38] Z. Zi, Sensitivity analysis approaches applied to system biology models, IET Syst. Biol. 5 (6) (2011) 336-346.

[39] J. Kirch, C. Tomaseth, A. Jensch, N. Radde, The effect of model rescaling and normalization on sensitivity analyis on an example of a MAPK pathway model, EPJ Nonlinear Biomedical Physics 4 (2016) 3.

[40] S. M. Blower, H. Dowlatabadi, Sensitivity and uncertainty analysis of complex models of disease transmission: an HIV model, as an example, International Statistical Review 62 (2) (1994) 229-243.

[41] S. Marino, I. B. Hogue, C. J. Ray, D. E. Kirschner, A methodology for performing global uncertainty and sensitivity analysis in systems biology, Journal of Theoretical Biology 254 (1) (2008) 178-196.

[42] D. Joseph, H. Bax, S. Karagiannis, Tumour-associated macrophage polarisation and re-education with immunotherapy, Frontiers in Bioscience 7 (2015) 334-351.

[43] A. Mantovani, F. Marchesi, A. Malesci, L. Laghi, P. Allavena, Tumourassociated macrophages as treatment targets in oncology, Nat. Rev. Clin. Oncol. 14 (7) (2017) 399-416.

[44] R. D. Boer, A. Perelson, Quantifying T lymphocyte turnover, J. Theor. Biol. 327 (2013) 45-87.

[45] D. Macallan, R. Busch, B. Asquith, Current estimates of T cell kinetics in humans, Current Opinion in Systems Biology 18 (2019) 77-86. 